\title{
Lithospheric failure on Venus
}

\author{
By A. C. FOWLER AND S. B. G. O'BRIEN ${ }^{1}$ \\ ${ }^{1}$ Mathematical Institute, 24-29 St Giles', Oxford OX1 3LB, UK \\ ${ }^{2}$ Department of Mathematics, University of Limerick, \\ Limerick, Republic of Ireland
}

Received 24 January 2002; accepted 10 October 2002; published online 29 August 2003

We develop a predictive model which has the ability to explain a postulated style of episodic plate tectonics on Venus, through the periodic occurrence of lithospheric subduction events. Present-day incipient subduction zones are associated with the existence of arcuate trenches on the Venusian lithosphere. These trenches resemble terrestrial subduction zones, and occur at the rim of coronae, uplift features thought to be due to deep-mantle convective plumes. The model we adopt represents the lithosphere as the thermal boundary layer which lies above a convective plume. We assume a temperature-dependent nonlinear viscoelastic rheology, and we assume a stress-based criterion for plastic yield. In developing this latter criterion, we are led to a re-interpretation of the strength envelope which is commonly used in analysing lithospheric stress, and we propose that the plastic yield strength has meaning (and is finite) below the lithosphere, using behaviour in the Earth as our "laboratory' justification for this view. An inferred yield stress on the Earth is $c a .300$ bar (30 MPa). Our model then shows that a thickening lithosphere becomes progressively more fluid as the stresses induced by the buoyant convective plume become large. Failure occurs when the effective lithosphere viscosity becomes equal to that of the underlying mantle. We show that reasonable expected values of yield stress in the range 100-200 bar (10-20 MPa) for Venusian mantle rocks are consistent within the framework of the model with radii of coronal trenches in the range 100-1200 km, and with the approximate time (200-800 Myr) which they may take to develop.

Keywords: Venus; subduction; yield stress; resurfacing

\section{Introduction}

Venus is a planet similar in size and, presumably, composition to the Earth, although it also has striking differences. Most notably, a dense $\mathrm{CO}_{2}$ atmosphere causes a greenhouse effect, which keeps the surface temperature at an alarming $750 \mathrm{~K}$. Despite this, there is (or was) a general expectation that the tectonic behaviour of Venus should be much like that of Earth.

The solid Earth consists of a refractory mantle of silicate rocks surrounding a dense iron-rich core (the outer part of which is liquid). This mantle convects because of its thermal buoyancy. Heating of the mantle is due both to cooling of the core and to the internal heating caused by radioactive decay of elements such as uranium and thorium. The motion itself is due to the creep of dislocations within the solid crystalline rock grains, and the rock thus behaves as an effectively viscous material, 


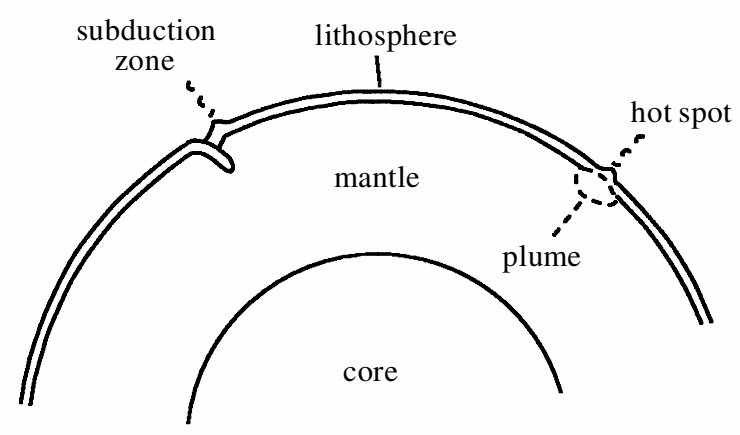

Figure 1. Cartoon of convection in the Earth's mantle.

with a viscosity that depends strongly on temperature (being more sticky where it is cold).

It is this convective motion which drives plate tectonics on the Earth, the 'plates' being the near-rigid segments of the Earth's surface that move due to the convection below; their rigidity is due to their relative coolness and consequent very high viscosity.

Figure 1 shows a cartoon of the convective style on Earth. The surface plates, also known as the lithosphere, sink into the mantle at subduction zones, where oceanic trenches exist, and new plate is created at mid-ocean ridges, which correspond to the upwelling parts of the convective circulation. Because the Rayleigh number of convection is high, the motion is thermally turbulent, and there are a consequent number of isolated thermal plumes. These can lead to partial mantle melting and consequent volcanism, for example at hot spots such as Hawaii or Iceland (which is also on the Mid-Atlantic Ridge).

The active plate-tectonic style of the Earth is evidenced particularly by its system of trenches and ridges, and an obvious question to ask is whether the other terrestrial planets have a similar convective style; the Moon, for example, does not, and its surface is therefore essentially as old as the Solar System itself.

What of Venus? The Pioneer, Venera, and, more recently, Magellan spacecraft missions sent back an enormous quantity of data, which revealed that Venus, although similar to Earth, has a very different tectonic history. Plate tectonics is apparently absent (Solomon et al. 1992), but impact crater distribution indicates that the different parts of the surface of the planet are of similar age, variously reckoned as being ca.500-1000 Myr (Schaber et al. 1992; Strom et al. 1994; Herrick 1994).

Nevertheless, Venus is a tectonically and convectively alive planet. There is much evidence of past volcanism, and there are numerous topographic features that indicate an actively convecting interior. Although the hypsometry of the planet is unimodal (unlike the bimodal continental/oceanic topography of Earth, indicating plate tectonics), there are highlands and lowlands; the latter are often taken to consist partly of large flood plains, while the highlands may be indicative of convective mantle upwellings. There are a large number of volcanic features, for example, dyke swarms as well as volcanoes, and channels, which may be the paths of extremely long lava flows. There are a large number of tectonic features indicating various kinds of tectonic deformation: tessera and wrinkle ridges are ridged features associated with tectonic deformation. Chasmata are long, linear to arcuate features consisting of deep troughs adjoining elevated ridges, while coronae are quasi-circular uplift features 


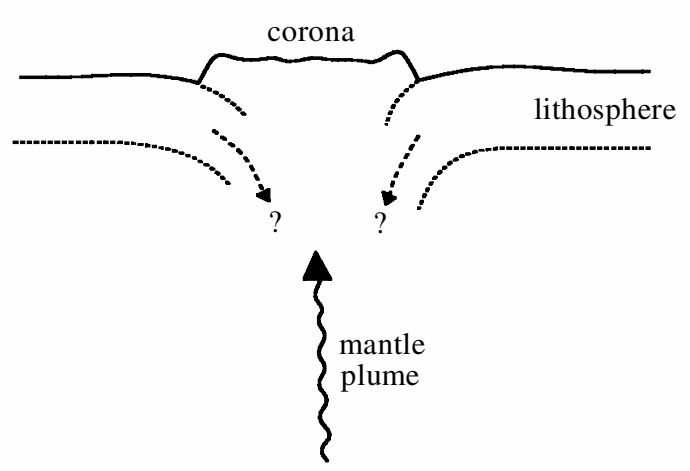

Figure 2. A cartoon of a lithosphere section through a Venusian corona, after Sandwell \& Schubert (1992). The surface looks much like that of a bubbling pan of milk, due to the arrival of a thermal plume from below. The diagram indicates the possibility of lithospheric collapse following plume impingement.

which may represent the surface expression of Venusian mantle plumes (Solomon \& Head 1991; Musser \& Squyres 1997) below a stagnant lithosphere. They consist of a central plateau surrounded by a raised rim and a moat. They have been described and visualized by Squyres et al. (1992), and classified by DeLaughter \& Jurdy (1999). A schematic section through a typical corona is indicated in figure 2. Reviews of some of the geology of Venus and descriptions of its inferred structure are provided by Philips \& Hansen (1994) and Nimmo \& McKenzie (1998).

We thus have a conundrum: Venus is actively convecting, but its surface indicates that the lithosphere is stagnant; on the other hand, its apparently uniform age of $500 \mathrm{Myr}$ suggests that this has not always been so. In order to explain this, Turcotte (1993) suggested that convection in Venus's mantle might be episodic in nature: a long period of stagnant lid convection (Solomatov \& Moresi 1996), in which the lithosphere thickens conductively, is interrupted by a catastrophic failure of the lid followed by overturning of the mantle, before resumption of the stagnant lid-type convection. The existence of subduction trench-like arcuate features at the rim of coronae (Sandwell \& Schubert 1992) is then a possible indication that the Venusian lithosphere is once again becoming unstable.

Coronae have typical radii in the range 100-300 km (DeLaughter \& Jurdy 1999). If we suppose that episodic subduction can occur, and that it is initiated after the impingement of a plume at the base of the lithosphere, we therefore need to seek a mechanism that can cause failure at distances of a typical order of $200 \mathrm{~km}$ from a central plume, at a time of the order of $500 \mathrm{Myr}$ after a previous overturning. The present paper is concerned with providing such a model.

The structure of the paper is as follows. In $\S 2$, we discuss the physical ingredients of our model. In particular, we address the difficulty within mantle-convection studies of describing subduction, and how this may be resolved by using a 'visco-plastic' rheology for mantle rocks, and we then extensively discuss the reasons such a rheology may be appropriate, and how it should be formulated. Section 3 then presents the mathematical model to be solved. There is a detailed mathematical analysis of this model, which occupies Appendix A, and which leads to a simplification of the model, which describes the evolution of the lithosphere by a free-boundary problem, following the arrival beneath it of a mantle plume. The numerical solution of this 
simplified model is described in $\S 4$ (the details of the numerical method being relegated to Appendix B), and a suite of numerical results are obtained. These describe the point and time of lithospheric failure (and consequent subduction) in terms of the prescribed physical parameters of the model. In $\S 5$, these results are discussed at length, and the conclusions of the study follow in $\S 6$.

\section{A physical model}

\section{(a) The initiation of subduction}

The problem of understanding how subduction occurs has been addressed by many authors (Turcotte et al. 1977; McKenzie 1977; Cloetingh et al. 1982, 1989; Mueller \& Phillips 1991; Shemenda 1992, Fowler 1993; Kemp \& Stevenson 1996), but is by no means conclusively resolved. The basic problem lies with the strength and rigidity of a relatively cold lithosphere, and most authors favour a mechanism for subduction consisting of failure of one type or another: either by formation of a thrust fault, or through (ductile) failure of the lithosphere. In particular, the latter mechanism is consistent with the concept of a 'plastic hinge' (Turcotte \& Schubert 1982), which can develop when the elastic core of the lithosphere disappears.

As Mueller \& Phillips (1991) remark, the problem of initiating subduction on a non-subducting planet such as Venus may be altogether different (and more difficult) than doing so on the Earth, where relative plate motions can allow mechanisms such as those persuasively studied by Shemenda (1992) to operate. In this paper we wish to develop a model previously suggested by Fowler (1993) and Fowler \& O'Brien (1996), and numerically validated by Moresi \& Solomatov (1995), which allows plastic failure of the cold lithosphere through the operation of the stresses which are generated by the underlying convection. In particular, no pre-existing convergent plate motion, such as envisaged by McKenzie (1977), is necessary for this mechanism to occur.

Our model is based on the following concept of variable viscosity convection. Mantle rocks such as olivine have an effective viscosity which varies very strongly with temperature. High-Rayleigh-number convection for such materials normally occurs as a rapid flow beneath a thick stagnant lid (Nataf \& Richter 1982; Moresi \& Solomatov 1995), which exists because the cold rocks towards the surface are extremely viscous. The existence of such a thick, cold, stagnant lid causes large buoyant stresses to be generated in the lid, and these can be of the order of kilobars, which is comparable to the breaking strength of crustal rocks.

We have previously suggested (Fowler \& O'Brien 1996) that lithospheric failure due to such high stresses might occur in the time-dependent mode envisaged for Venus. Specifically, we analysed variable (Newtonian) viscosity convection in a twodimensional Cartesian geometry with horizontal coordinate $x$ and vertical (downwards) depth coordinate $z$, and showed that, as time develops, a thick, stagnant lid $z=s(x, t)$ grows downwards, but that a plastically failing zone $0<z<q(x, t)$ may also occur, whose thickness grows more rapidly. At some critical time and at some distance from the upwelling, the plastic zone reaches the base of the lithospheric lid (see figure 3), and at this value of $x$ the effective viscosity of the whole vertical depth of the lithosphere is reduced to that of the convecting mantle below, and subduction is hypothesized to begin.

Our aim in this paper is to examine this hypothesis for the onset of subduction in the context of the suggestion by Sandwell \& Schubert (1992) (see also Schubert et 


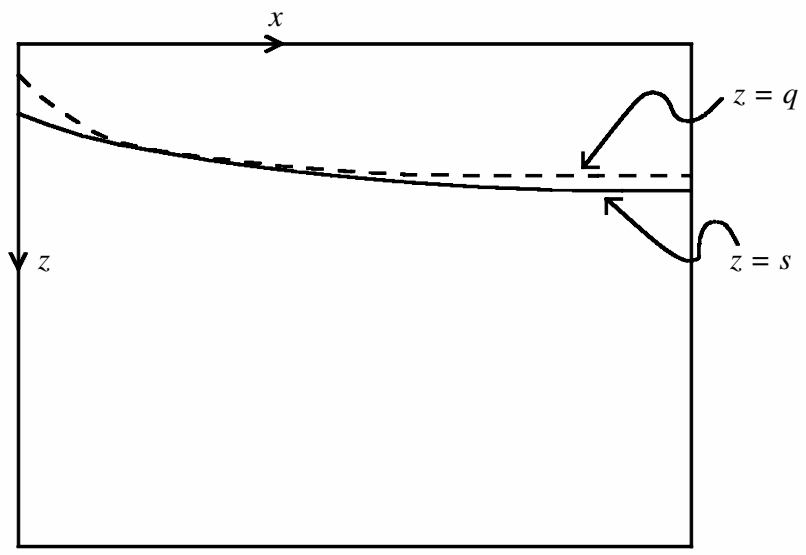

Figure 3. The way in which the base of the plastic zone at $z=q$ reaches the lithosphere base at $z=s$, causing failure and subduction at the point of tangency. This cartoon is appropriate for a Newtonian rheology, but we shall find later that, for a non-Newtonian rheology, failure occurs before the plastic zone reaches the base of the lithosphere.

al. (1994)) that arcuate features on certain Venusian coronae resemble subduction trenches on the Earth. We thus envisage a thermal plume in the rapidly convecting Venusian mantle which drives a radial flow outward from the corona beneath the lithosphere. This flow induces large stresses, and we seek to study whether these stresses are sufficient to cause plastic failure in the way suggested by Fowler \& O'Brien. Apart from the posing of this problem in the cylindrical geometry which is relevant to the sub-lithospheric flow outwards from a plume, other developments of the earlier theory are that: we include viscoelasticity in the model; we treat the viscoelastic-plastic transition more realistically than in our previous paper; we consider the effect of radioactive heating in the model; and we allow for stress dependence in the viscous part of the rheology. In particular, the stress dependence of the flow law has a dramatic quantitative effect on the criterion for failure, and in fact failure will occur before the plastic zone reaches the base of the lithosphere: this is because the elevated stresses in the lithosphere cause a marked reduction of the viscosity below that at the same temperature for a Newtonian fluid.

\section{(b) Rheology of the mantle}

Viscous flow of the mantle becomes effective at temperatures greater than $c a .1000 \mathrm{~K}$, and is generally assumed to be given by a creep law in the form

$$
\dot{\varepsilon}=A \tau^{n} \exp \left[-E^{*} / R T\right]
$$

(Kirby \& Kronenberg 1987), where $n \approx 3.5$ for dislocation creep, $T$ is the temperature, $R$ is the gas constant, and $E^{*}$ is the activation energy, usually (e.g. for olivine) a large quantity, so that the strain rate $\dot{\varepsilon}$ varies very strongly with temperature. The variation with stress $\tau$ is significant, though less pronounced. Although creep experiments agree on this form of the viscous rheology, it needs to be realized that applying it to the Earth requires an extrapolation in strain rates of many orders of magnitude. One needs to be wary of this, particularly at lower temperatures, where long-term stress weakening may cause higher rates of flow than (2.1) would suggest. 
In particular, several authors (e.g. Kirby 1977; Tsenn \& Carter 1987) have suggested that, in effect, $n$ should increase at higher stresses or lower temperatures.

At temperatures below 700-800 K, and for short-time-scale phenomena (e.g. seismic waves), the mantle behaves elastically, and it is often convenient to model the combined viscoelastic behaviour by the Maxwell model, which is most simply represented by the equation

$$
\dot{\varepsilon}=\frac{\tau}{2 \eta}+\frac{\dot{\tau}}{E_{\mathrm{M}}},
$$

where the overdot denotes a time derivative, $\eta$ is the effective viscosity

$$
\eta=\frac{1}{2 A \tau^{n-1}} \exp \left(-\frac{E^{*}}{R T}\right),
$$

and $E_{\mathrm{M}}$ is an appropriate elasticity modulus (proper tensorial forms are described below). Inspecting (2.2), we see that there is an intrinsic Maxwell time

$$
t_{\mathrm{M}}=\frac{2 \eta}{E_{\mathrm{M}}},
$$

and that for $t \ll t_{\mathrm{M}}$, the behaviour is elastic, but for $t \gg t_{\mathrm{M}}$, it is viscous.

Further, $t_{\mathrm{M}}=t_{\mathrm{M}}(\tau, T)$ depends (through $\eta$ ) on stress and temperature, and in particular is a strong function of temperature: in the mantle, this implies that the region where the jump between viscous and elastic behaviour occurs is fairly sharp.

\section{(c) Failure}

Crystalline mantle rocks fail if subjected to sufficiently high tensile or compressive stresses. At low confining pressures, such failure is said to be brittle and occurs by the formation of cracks. It is usually assumed in the mantle that brittle failure is described by Byerlee's law (Byerlee 1978; Brace \& Kohlstedt 1980), although Byerlee was in fact concerned with rock friction. Byerlee's law is essentially of the form

$$
\tau_{\mathrm{f}}=K p,
$$

where $p$ is the lithospheric pressure, and $\tau_{\mathrm{f}}$ is the failure stress; the number $K \sim 1$ for tensile failure. (A similar failure criterion holds for compressive failure with a larger value of $K$.) Since the lithostatic pressure gradient in the Earth or Venus is ca. $0.3 \mathrm{kbar} \mathrm{km}^{-1}$, Byerlee's law suggests a failure stress of $c a .10 \mathrm{kbar}$ at a depth of $30 \mathrm{~km}$. In fact, other mechanisms become operative at such depths.

Brittle failure is associated with the formation and coalescence of microcracks (Evans et al. 1990), but at higher temperatures, plasticity prevents coalescence and, at higher temperatures still, microcracking does not occur. Instead, strain/stress tests (at a fixed strain rate) indicate that the strain $\varepsilon$ increases with stress $\tau$ until ductile failure occurs at the yield stress $\tau_{\mathrm{d}}$. Essentially, the strain then continues to increase without bound and without further increase in stress. The term ductile failure in this case refers to the onset of viscous creep behaviour, and can be described in terms of the Maxwell model of (2.2): at a constant strain rate $\dot{\varepsilon}$, the solution of (2.2) (for simplicity we use $n=1$ for illustration) is

$$
\tau=\tau_{\mathrm{d}}\left[1-\exp \left\{-E_{\mathrm{M}} \frac{\varepsilon}{\tau_{\mathrm{d}}}\right\}\right],
$$


where the ductile yield stress is just the viscous stress $\tau_{\mathrm{d}}=2 \eta \dot{\varepsilon}$. In general, the ductile yield stress depends strongly on temperature, and also on the deformational time-scale $\dot{\varepsilon}^{-1}$.

In fact, it is somewhat confusing to describe $\tau_{\mathrm{d}}$ above as a yield stress. The confusion is enhanced by the evident fact that laboratory experiments at constant strain rate will yield stress/strain formulae similar to (2.6) whether viscous creep or plastic failure is occurring.

It is also difficult to extrapolate laboratory experimental results to high temperatures and pressures (Karato \& Wu 1993), and in particular, it is hazardous to do so in lithospheric conditions, where the corresponding strain rates are much smaller than can be measured. However, the Earth itself acts as a laboratory which can guide us in assessing rheological behaviour, in three separate ways.

There are three ways to describe the rheology of continua: as viscous fluids, as elastic solids, or as plastic materials. These descriptions can be combined, as in viscoelastic fluids, or elastic-plastic solids. We discuss some of these descriptions below. The first point to make is that deformation in the lithosphere is accompanied by the formation of fractures, for example, those which accompany earthquake slip on major transform faults, such as the San Andreas fault, or intraplate fracture zones. It is evident from this fact that a viscous or an elastic (or viscoelastic) rheology is not wholly appropriate. The presence of such features suggests that at low temperatures and elevated stresses, continuous (on a geological time-scale) deformation occurs via stick-slip motion, for which the natural model is that of a plastic material with a yield stress. In common with classical models for such materials, it is natural to propose that deformation at lower stresses is described by a viscoelastic rheology.

Secondly, an estimate for the likely magnitude of such a yield stress is provided by the observations of deep earthquakes in the Benioff zones of subducting slabs. If a mean temperature difference of $\Delta T$ exists across a subducting slab of thickness of thickness $d_{\mathrm{s}}$, then an estimate for the yield stress $\tau_{\mathrm{c}}$ is

$$
\tau_{\mathrm{c}}=\frac{1}{2} \alpha \rho_{\mathrm{a}} g d_{\mathrm{s}} \Delta T
$$

(the factor of two is used because the stress is supported on both sides), where $\alpha$ is the thermal expansion coefficient, $\rho_{\mathrm{a}}$ is the density, and $g$ is the acceleration due to gravity. An upper limit is provided by a surface lithosphere thickness of $100 \mathrm{~km}$, and a mean temperature difference from surface to asthenosphere (averaged across the lithosphere) of $600 \mathrm{~K}$. If we take $\alpha=3 \times 10^{-5} \mathrm{~K}^{-1}, \rho_{\mathrm{a}}=3.3 \times 10^{3} \mathrm{~kg} \mathrm{~m}^{-3}$ and $g=10 \mathrm{~m} \mathrm{~s}^{-2}$, then this suggests $\tau_{\mathrm{c}} \lesssim 300$ bar. An independent lower bound can be obtained through observed stress drops in earthquakes. Typically, these are of similar magnitude (Henry et al. 2000). Independent estimates of the state of the stress in the lithosphere (England \& Molnar 1991; Von Herzen et al. 2001) are also in the range 250-400 bar.

Thirdly, direct measurements of continental collision and orogeny (England \& Molnar 1997; Davies et al. 1997) are consistent with viscous lithospheric flow with viscosities of the order of $10^{22} \mathrm{Pas}$. This is much lower than any experimentally extrapolated continuous flow law would suggest at low temperatures, even at elevated stresses, but is consistent with the application of a visco-plastic flow law, as we suggest here.

If such stress induced failure controls the long-term fluidity of the lithosphere, then it is likely that the yield envelope (discussed below) constructed from the brittle and 
ductile yield curves overestimates the strength at depth (Karato \& Wong 1995), at least over long time-scales. The implication is that the Byerlee law, which may be appropriate for short time-scale strength estimates at shallow depths, is inapplicable to the mantle-convection problem under discussion.

\section{(d) Elastic-perfectly plastic materials}

Plastic failure (in metals, for example) is commonly associated with the attainment of a fixed critical value of the stress, determined for example by the Tresca or Von Mises yield criterion. Such perfectly plastic materials can be interpreted in terms of viscous creep laws by supposing that the viscous creep law $\dot{\varepsilon}=f(\tau)$ also saturates at a value $\tau=\tau_{\mathrm{c}}$, the perfectly plastic yield stress: thus $\dot{\varepsilon}=\tau / 2 \eta$ for $\tau<\tau_{\mathrm{c}}$, and $\dot{\varepsilon}=\tau_{\mathrm{c}} / 2 \eta_{\mathrm{c}}$ for $\tau=\tau_{\mathrm{c}}$. Here $\eta=\eta_{\mathrm{c}}$ when $\tau=\tau_{\mathrm{c}}$; a rigid-plastic medium would be one for which $\dot{\varepsilon}=0$ for $\tau<\tau_{\mathrm{c}}$.

Although we can interpret elastic-plastic behaviour in terms of a viscoelastic law, it is not obvious that this is consistent with the classical treatment of such materials. For purely elastic deformations, the strain increment in tensor form is

$$
\mathrm{d} \varepsilon_{i j}^{\mathrm{e}}=\frac{\mathrm{d} \tau_{i j}}{2 \mu}+\frac{(1-2 \sigma)}{E} \mathrm{~d} \sigma_{k k} \delta_{i j},
$$

where the superscript 'e' refers to elastic deformation, the summation convention is used, $\delta_{i j}$ is the Kronecker delta, $\sigma$ is Poisson's ratio, $\mu$ is the shear modulus, $E$ is Young's modulus, $\sigma_{i j}$ is the stress tensor and $\tau_{i j}=\sigma_{i j}-\frac{1}{3} \sigma_{k k} \delta_{i j}$ is the deviatoric stress.

In an ideal plastic material (Hill 1950), we suppose that beyond failure the strain is the sum of an elastic increment and a plastic increment $\mathrm{d} \varepsilon_{i j}^{p}$, which is assumed parallel to the deviatoric stress (so there is no plastic volume change); thus we have the Prandtl-Reuss equations

$$
\mathrm{d} \varepsilon_{i j}=\tau_{i j} \mathrm{~d} \Omega+\mathrm{d} \varepsilon_{i j}^{\mathrm{e}},
$$

where $\Omega$ is a Lagrange multiplier, which is determined by the additional yield criterion. For example, the Von Mises criterion constrains the stress to lie on the hypersurface

$$
\tau_{i j} \tau_{i j}=2 \tau_{\mathrm{c}}^{2}
$$

How does this model relate to the Maxwell model? In incremental, tensorial form, we would write (2.2) (noting (2.8)) as

$$
\mathrm{d} \varepsilon_{i j}=\frac{\tau_{i j}}{2 \eta} \mathrm{d} t+\mathrm{d} \varepsilon_{i j}^{\mathrm{e}},
$$

and we see that in conditions where a strain rate is defined, the two models are identical, with $\mathrm{d} \Omega=\mathrm{d} t / 2 \eta$.

The above discussion gives us some confidence that a Maxwell-type model can be used to describe both viscous and elastic behaviour and also, if necessary, Von Misestype yield (if the stresses are materially bounded). In the mantle, however, rockdeformation experiments (Goetze \& Evans 1979) apparently indicate that creep continues to very high stresses: (2.1) applies for $\tau<2000$ bar, and beyond this $\dot{\varepsilon}$ increases more rapidly, but a limiting yield stress does not seem to specifically occur. 


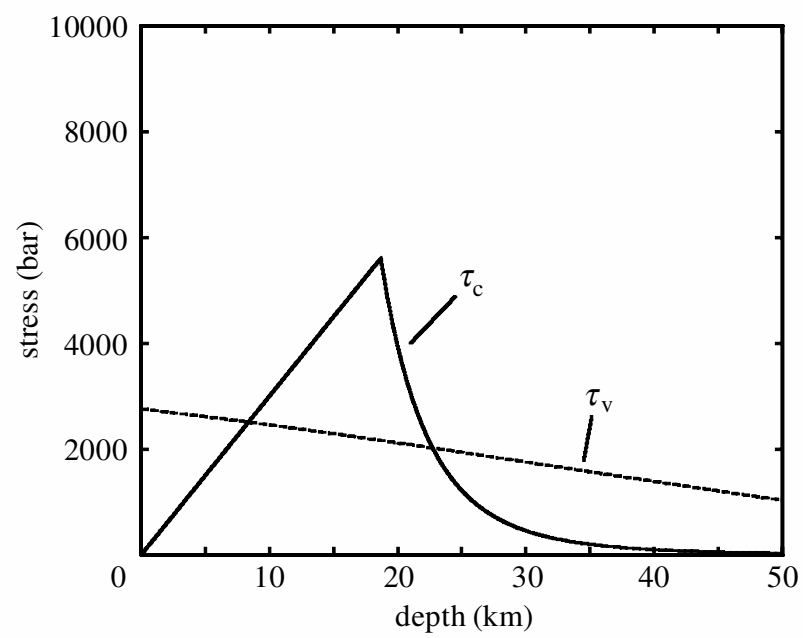

Figure 4 . Brittle and ductile yield stresses $\left(\tau_{\mathrm{c}}\right)$, calculated using parameter values in the text, for a representative Venusian lithosphere temperature. Also shown is a representative lithospheric shear stress $\left(\tau_{\mathrm{v}}\right)$ calculated from results in Fowler (1985) (for a purely viscous mantle).

As we have discussed above, there are difficulties in distinguishing between creep and failure in constant-strain-rate tests, and also in extrapolating such results to mantle conditions. In this paper, we will suppose that ductile failure does occur (as opposed to ductile yield, i.e. creep), and that the failure criterion is represented by a relation of Von Mises type. This suggestion is not new and has been made before (Hobbs \& Ord 1988).

\section{(e) The yield envelope}

(Plastic) failure in the Earth is often supposed to be brittle (described by Byerlee's law) or ductile, the latter being defined by the stress at which the (sharp) switchover between viscous and elastic behaviour occurs in the lithosphere. From (2.1) and (2.2), this is defined (for a power-law rheology) by

$$
A \tau^{n} \exp \left[-\frac{E^{*}}{R T}\right]=\frac{\tau}{E_{\mathrm{M}} t_{\mathrm{d}}},
$$

where the deformational time-scale $t_{\mathrm{d}}=\dot{\varepsilon}^{-1}$ is prescribed. For $n>1,(2.12)$ defines a ductile yield envelope $\tau=\tau_{\mathrm{d}}(T, \dot{\varepsilon})$ as shown in figure 4 . Since $\tau_{\mathrm{d}} \propto \exp \left[E^{*} /(n-1) R T\right]$ and $T$ increases with depth and thus pressure, $\tau_{\mathrm{d}}$ is a (rapidly) decreasing function of depth. On the other hand, the brittle yield stress $\tau_{\mathrm{f}}$ increases with depth, more or less linearly, and this gives the familiar brittle-ductile failure envelope in figure 4 .

The interpretation of these curves is as follows: if $\tau>\tau_{\mathrm{f}}$, brittle yield occurs, while if $\tau>\tau_{\mathrm{d}}$ the behaviour is essentially viscous. For $\tau<\tau_{\mathrm{f}}$ and $\tau<\tau_{\mathrm{d}}$, the material is elastic. Fowler (1985) showed that in the viscous lid of a convecting mantle of strongly temperature-dependent viscosity, very high tensile stresses develop, as schematically shown in figure 4. If we suppose that these stresses also develop in the equivalent viscoelastic mantle, then the implication for the lithosphere is that, in practice, there would be an elastic core lying below a fractured layer (subject to brittle failure) and above a viscously creeping mantle. 
There is a logical inconsistency in the interpretation of the ductile yield curve in figure 4 , in that it assumes a prescribed value of the time-scale (here $10^{16} \mathrm{~s}$ ), whereas in fact the strain rate depends on the viscosity, and decreases rapidly with decreasing temperature. We consider that a better approach to providing a rheological description of the lithosphere is to couple the viscoelastic model described above with a plastic failure stress, which describes long-term continuous deformation through short-term occasional slip events (earthquakes). We call the resulting yield envelope the 'deformational yield envelope', to distinguish it from the brittle-ductile yield envelope portrayed in figure 4 .

It is this situation that we attempt to describe in this paper. We anticipate the existence of a failed zone at the surface, and perhaps an elastic core beneath it. Total lithospheric failure is then deemed to occur if and when the elastic core shrinks to zero, and if the resultant effective viscosity of the lithosphere becomes comparable to that of the underlying mantle. (The reason for this second constraint is that, even if the elastic core disappears, the viscous lid will remain immobile if it is viscous enough.) In the application of the model to Venus, we find that the elevated surface temperature implies that the elastic core may not actually be present (if we consider only long time-scale deformation).

\section{(f) Modelling the lithosphere}

Modelling elastic-plastic behaviour on the Earth is complicated by the existence of the weak crust, which has its own (deformational) yield envelope. This complication may be unnecessary on Venus, where the lack of water may imply that the crust is relatively strong (Mackwell et al. 1998). At any rate, we will confine our attention to the assumption of a single lithospheric constituent with a single deformational yield envelope.

We anticipate a zone of failure at the surface. We suppose that the shear stress is then pinned to the yield value, and we suppose that strains are parallel to stresses; that is, we model the failure zone as a perfectly plastic material with a Von Mises or Tresca yield criterion.

Suppose there is an underlying elastic core. The failed zone is then effectively elastic, with an unknown effective shear modulus which is determined by the requirement that the stress is equal to the failure value. We might model the ductile yield line at the base of the elastic core as a sharp boundary between viscous and elastic behaviours, so that the relevant boundary condition on the viscous lithosphere beneath would be a no-slip condition. Since the stresses in the underlying viscous lid are still high, it is also quite possible that an elastic core does not exist. On Venus, this is in fact quite likely, because of the high surface temperature. In that case, the failed region also behaves effectively viscously, and our model is then essentially that which we used before (Fowler \& O'Brien 1996).

To summarize the above discussion: the simplest, most appropriate rheology that we can use to describe the behaviour of the lithosphere throughout its thickness is that of a Maxwell viscoelastic material having a yield surface of Tresca type. Two possible lithospheric zones can then occur: a brittle surface zone, and a ductile asthenosphere, separated (or not) by an elastic core. Because of the elevated temperatures on Venus, we make a simplifying assumption that the elastic core is absent, and we can check a posteriori that this is a reasonable assumption. The effective 


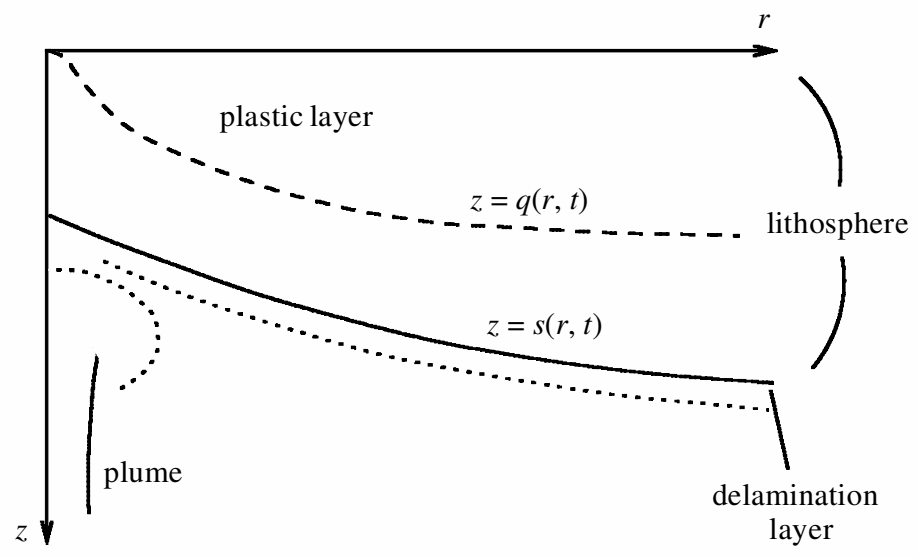

Figure 5. Geometry of the model flow.

rheology is then in fact viscoplastic, in the sense that we defined earlier (Fowler \& O'Brien 1996). Nevertheless, lithospheric failure requires a further condition to occur, namely that the effective viscosity in the failure zone decrease to the ambient asthenospheric value. This is in line with our previous discussion.

\section{Mathematical model}

We consider slow (that is, high Prandtl number) convection of the Venusian mantle. We model the rheology of the mantle by a strongly temperature-dependent viscoelastic Maxwell material, as described in $\S 2$. Specifically, we anticipate that the elastic term of this model will only be of significance in the (stagnant) lithosphere, where strains and strain rates are small, and in consequence we can avoid the rigours of frame-indifferent material derivatives (Fowler 1997, p. 111), and assume a tensorial constitutive law of the form (from (2.8) and (2.11))

$$
\tau_{i j}=2 \eta\left[\dot{\varepsilon}_{i j}-\frac{1}{2 \mu} \frac{\partial \tau_{i j}}{\partial t}+\frac{(1-2 \sigma)}{3 E} \frac{\partial p}{\partial t} \delta_{i j}\right],
$$

where

$$
\frac{1}{2 \eta}=A \tau^{n-1} \exp \left[-\frac{E^{*}}{R T}\right],
$$

$\eta$ is the shear viscosity, $E$ is Young's modulus, $\mu$ is the shear modulus and $\sigma$ is Poisson's ratio. We will also suppose that a yield stress exists, beyond which either the longitudinal stress or the second stress invariant cannot be taken. The first of these corresponds to the Tresca criterion, the second is Von Mises's criterion. As relentlessly discussed in $\S 2$, a separate explicit description of deformation on the plastic yield envelope is not necessary, as this is automatically included in the viscoelastic rheology.

We now consider a cylindrically symmetric flow centred at a mantle plume. We take coordinates $z$ downwards and $r$ radially outwards, and corresponding velocity components are $w$ and $u$; the geometry is that of figure 5 (with $r$ replacing $x$ ). In 
component form, the Maxwell rheology (3.1) is

$$
\left.\begin{array}{l}
\tau_{r r}=2 \eta\left[\frac{\partial u}{\partial r}-\frac{1}{2 \mu} \frac{\partial \tau_{r r}}{\partial t}+\frac{(1-2 \sigma)}{3 E} \frac{\partial p}{\partial t}\right], \\
\tau_{z z}=2 \eta\left[\frac{\partial w}{\partial z}-\frac{1}{2 \mu} \frac{\partial \tau_{z z}}{\partial t}+\frac{(1-2 \sigma)}{3 E} \frac{\partial p}{\partial t}\right], \\
\tau_{r z}=\eta\left[\frac{\partial u}{\partial z}+\frac{\partial w}{\partial r}-\frac{1}{2 \mu} \frac{\partial \tau_{r z}}{\partial t}\right],
\end{array}\right\}
$$

with the deviatoric hoop stress being $\tau_{\theta \theta}=-\left(\tau_{r r}+\tau_{z z}\right)$. The Boussinesq equations for the velocity field are then

$$
\left.\begin{array}{rl}
\frac{1}{r} \frac{\partial}{\partial r}(r u)+\frac{\partial w}{\partial z} & =0, \\
\frac{\partial p}{\partial r} & =\frac{1}{r} \frac{\partial}{\partial r}\left(r \tau_{r r}\right)+\frac{\partial \tau_{r z}}{\partial z}+\frac{\tau_{r r}+\tau_{z z}}{r}, \\
\frac{\partial p}{\partial z} & =\frac{1}{r} \frac{\partial}{\partial r}\left(r \tau_{r z}\right)+\frac{\partial \tau_{z z}}{\partial z}+\rho_{\mathrm{a}}\left[1-\alpha\left(T-T_{\mathrm{a}}\right)\right] g,
\end{array}\right\}
$$

where we take the gravitational acceleration $g$ to be constant, and suppose the mantle density is $\rho=\rho_{\mathrm{a}}\left[1-\alpha\left(T-T_{\mathrm{a}}\right)\right]$; here, $\rho_{\mathrm{a}}$ is a reference value at temperature $T_{\mathrm{a}}$, which we shall take to be the value below the lithosphere, and $\alpha$ is the thermal expansion coefficient, assumed constant. The viscosity $\eta$ in (3.3) is given by (2.3), where the second stress invariant $2 \tau^{2}$ is defined by

$$
2 \tau^{2}=2 \tau_{r z}^{2}+\tau_{r r}^{2}+\tau_{z z}^{2}+\left(\tau_{r r}+\tau_{z z}\right)^{2} .
$$

Finally, the temperature $T$ satisfies

$$
\frac{\partial T}{\partial t}+u \frac{\partial T}{\partial r}+w \frac{\partial T}{\partial z}=\kappa \nabla^{2} T+Q
$$

where $\kappa$ is the thermal diffusivity, assumed constant, and $Q$ is the volumetric heat release due to radioactive decay processes.

\section{(a) Boundary conditions}

We visualize a situation where these equations describe the lithospheric dynamics above an actively convecting mantle, but where the radial flow is generated by the arrival of a mantle plume. Below the lithosphere, it is appropriate for $T$ to tend to $T_{\mathrm{a}}$, a constant, while the deviatoric stresses become small relative to their lithospheric values: in particular, we can effectively prescribe $\tau_{r z} \rightarrow 0$ below the lithosphere.

At the surface $z=0$, we prescribe the surface temperature $T=T_{\mathrm{s}}$, and also $\tau_{r z}=0$, although in line with our previous discussion, we do in fact anticipate the existence of a failure zone $0<z<q(r, t)$ in which a failure criterion of Von Mises type would imply

$$
\tau=\tau_{\mathrm{c}}
$$

where $\tau_{\mathrm{c}}$ is the yield stress. We will take $\tau_{\mathrm{c}}$ as constant, but it is simple to allow it to depend on depth, for example. Below this, we assume an effectively viscous 
stagnant lithosphere $q<z<s(r, t)$ (the assumption will be validated if the time derivative terms in (3.3) are negligible). At $z=s$, there is a thin delamination layer, which couples the sticky lid to the mobile asthenosphere, and through which the temperature gradient changes sharply. This description is equivalent to that of Fowler (1993) and Fowler \& O'Brien (1996).

\section{(b) Model simplification}

A detailed analysis of this problem is presented in Appendix A. There we show that a suitably non-dimensionalized version of the model admits various asymptotic simplifications, and in particular, we show that the thermal structure of the lithosphere and its depth is determined from the following set of equations, in which the variables and parameters are dimensionless.

The lithospheric temperature satisfies (A 27), that is

$$
\left.\begin{array}{rl}
T_{t} & =T_{z z}+h, \\
T & =T_{0} \quad \text { on } z=0, \\
T & =1, \quad T_{z}=\Gamma \quad \text { on } z=s .
\end{array}\right\}
$$

Note that $\Gamma$ is not known, but that if $s$ is determined, then $\Gamma$ can be found; thus $\Gamma$ is functionally dependent on $s$. The unknown lithosphere base $z=s(r, t)$ is then solved from the delamination layer equation (A 36),

$$
\omega^{3}\left(\frac{r s^{\prime}}{\Gamma^{3}}\left|\frac{s^{\prime}}{\Gamma}\right|^{n-1}\right)^{\prime}=B_{n} r \Gamma
$$

where $B_{n}$ is shown in figure 14; (3.9) is effectively an extra boundary condition for the free boundary $s(r, t)$. The boundary conditions for (3.9) are discussed in the following section. Finally, the plastic lid base (the ductile-brittle transition layer) is at $z=q(r, t)$, computed from (A 82):

$$
2\left(r^{1 / 2} q\right)^{\prime}=-\frac{r^{1 / 2}}{C} \int_{0}^{s} z T_{r} \mathrm{~d} z .
$$

This equation suggests that $q$ grows roughly like $s^{2}$, and thus will eventually 'catch up' with $s$; as $q / s$ increases, the effective viscosity of the lithosphere progressively decreases, and our object is to compute when it is sufficiently weak that it will sink into the mantle. The parameters $h, \omega, B_{n}$ and $C$ are prescribed in Appendix A, specifically in (A 14), (A 18), (A 40) and (A 59).

\section{Plume dynamics and lithosphere failure}

(a) Boundary conditions

In order to solve (3.8) with (3.9), we require boundary conditions to be specified for $s$. These involve a prescription of how the mantle plume, which we suppose is emplaced at $r=0$, affects (and indeed causes) the flow. First we note that there is a perfectly valid solution if $\Gamma=0$, when $s=s(t)$, and (3.8) is analogous to a (CrankGupta type) free-boundary problem. This corresponds to a freely growing conductive 
lithosphere, without convection beneath. This eventually becomes unstable to a largescale circulatory flow, but we imagine that convective instability of the hot lower boundary, and subsequent plume development, will be more rapid.

One boundary condition follows from consideration of (A 33), which indicates that the stream function $\Psi$ in the delamination layer is proportional to $r s^{\prime n} / \Gamma^{n+2}$ (assuming $s^{\prime}>0, \Gamma>0$ as we subsequently find), and in order that $\Psi \rightarrow 0$ as $r \rightarrow 0$ as we require, we must specify the stronger condition

$$
s_{r}=0 \quad \text { at } r=0,
$$

providing $\Gamma$ is finite at $r=0$ (which will be the case if $s>0$ there).

The second condition that we apply is to specify $s$ at $r=0$ :

$$
s=s_{0}(t) \quad \text { at } r=0 .
$$

Imagine that a cooling lithosphere grows (uniformly) from an overturning at an earlier time $t=-t_{0}$. At time $t=0$ (when $s=s_{00}$ ), a plume head arrives below the lithosphere, at $r=0$. The plume delivers a heat flux to the lithosphere, which we might characterize by prescribing $\Gamma$ at $r=0$, and (we suppose) it causes a corona to form, which we might describe by prescribing the uplift at $r=0$. Clearly, prescription of $\Gamma$ in (3.8) at $r=0$ allows the determination of $s=s_{0}$ there, and vice versa. Therefore, the effect of the plume can equally well be described by prescribing $s=s_{0}$ at $r=0$. This is actually preferable, because the uplift result (A 64) can be used to infer an estimate for $s_{0}$ based on observations of actual coronal plateau elevations. In (A 26), we have $\alpha T_{\mathrm{a}} d \approx 5 \mathrm{~km}$. For a typical plateau elevation of $h_{\mathrm{s}}=2 \mathrm{~km}$, this indicates $\Delta_{\mathrm{s}}-\Delta_{\mathrm{s}}^{\infty} \approx 0.4$ (where $\Delta_{\mathrm{s}}^{\infty}$ is the uplift far from the plume) and if we suppose a linear temperature profile, so that (A 64) gives $\Delta_{\mathrm{s}}-\Delta_{\mathrm{s}}^{\infty}=\frac{1}{2}\left(1-T_{0}\right)\left(s_{00}-s_{0}\right)$, then a plume indentation of magnitude

$$
s_{00}-s_{0} \approx \frac{2 h_{\mathrm{s}}}{\alpha\left(T_{\mathrm{a}}-T_{\mathrm{s}}\right) d}
$$

is indicated and this is about 1.4 for $h_{\mathrm{s}}=2 \mathrm{~km}$. Evidently, plumes can cause serious indentation of the thermal lithosphere. An alternative interpretation is that plume delivery halts growth of the lithosphere locally, so that uplift is caused as the lithosphere away from the plume head continues to grow.

\section{(b) Failure}

The numerical method of solution of (3.8) and (3.9) is described in Appendix B; it yields the functions $T(z, r, t), s(r, t)$ and $q(r, t)$. The latter is computed, using (3.10), in the form

$$
q=\frac{1}{2 C r^{1 / 2}} \int_{0}^{r} r^{1 / 2}\left[s^{2}\left\{\frac{1}{2}-\int_{0}^{1} \eta T \mathrm{~d} \eta\right\}\right]^{\prime} \mathrm{d} r .
$$

Near $r=0,($ B 12) implies

$$
s \approx s_{0}+\frac{\beta \Gamma_{0} n}{n+1}\left(\frac{\Gamma_{0}^{3}}{2}\right)^{1 / n} r^{1+(1 / n)}
$$

while from (3.10) we have that

$$
q=O\left(r^{(n+1) / n}\right)
$$


Therefore, $q<s$ for small $r$, and also (3.10) implies that $q=0$ at $t=0$ (since $T_{r}=0$ then). Using a linear interpolation between grid points, we can therefore compute

$$
T_{q}(r, t)=T(q(r, t), r, t),
$$

and $T_{q}=T_{0}$ for $r=0$ and $t=0$. Failure is deemed to occur when the effective viscosity at $z=q$ becomes equal to that at $z=s$, which is $\eta=O(1)$. Equating the right-hand side of (A 74) to unity defines a critical value of $T_{q}$ as

$$
T_{q}=\left(1+2(n-1) \varepsilon \ln \frac{1}{\varepsilon}+\varepsilon \ln \left[\frac{2 \nu^{2}|U| T_{q}^{\prime}}{C T_{q}^{2}}\right]\right)^{-1},
$$

and, denoting this critical value as $T_{\mathrm{c}}$, we may approximate it as

$$
T_{\mathrm{c}}=\frac{1}{1+2(n-1) \varepsilon \ln (1 / \varepsilon)} .
$$

For values $n=3.5$ and $\varepsilon=0.027, T_{\mathrm{c}} \approx 0.67$. Since for small $h, T$ approaches a linear profile (in fact, quite rapidly), we see that failure will occur when, approximately,

$$
\frac{q}{s}=\frac{1-T_{0}}{T_{\mathrm{c}}-T_{0}},
$$

and for Venus (with $T_{\mathrm{a}}=1700 \mathrm{~K}$ ) this is $q / s \approx 0.41$. The dependence on $n$ is important, since failure requires $q / s=1$ for $n=1$, for example. In fact, failure will occur immediately for large enough $n$. For Venus, this value is about 7.5, and this is consistent with numerical computations which suggest the same thing (Weinstein \& Olson 1992).

One might argue that failure will actually happen somewhat before this, since numerical studies indicate that lid motion actually begins when the viscosity contrast across the lid is about $10^{4}$. This would be effected in (4.8) by adding a term $\varepsilon \ln \eta_{\mathrm{c}}$ to the denominator, where $\eta_{\mathrm{c}}$ is the viscosity contrast at failure. With the choice $\eta_{\mathrm{c}}=10^{4}$, for example, this would change the critical value of $T_{\mathrm{c}}$ to about 0.57 , and failure would be deemed to occur earlier. On the other hand, one might argue that the resultant high-viscosity lid would nevertheless take a long time to founder.

\section{(c) Length-scales and parameter choices}

The solution of the problem depends on the five internal parameters $n, T_{0}, h, \beta$ and $C$, as well as the initial and boundary data for $s$. In order to present the simplest picture, we choose the initial and boundary data for $s$ to be the same, that is we specify

$$
s=s_{0} \text { (const.) at } t=0 \text { and } r=0,
$$

so that the solutions depend also on the one additional parameter $s_{0}$. Since a proper description of the effect of the plume head on the boundary condition at $r=0$ is not available, this seems reasonable. It implies that when a plume reaches the lithosphere, it prevents further growth of the lithosphere there without any additional erosion.

Suppose that failure occurs when $T_{q}$ first reaches $T_{\mathrm{c}}$ at a value $r=r^{*}$ when $t=t^{*}$. These values depend on the five dimensionless parameters $T_{0}, h, \beta, C$ and $s_{0}$. We take $T_{0}$ to be fixed, but wish to consider variations of the other parameters. To do 
so, it is convenient to write the definitions of these parameters in terms of various intrinsically defined length-scales. Our aim in doing so is to remove the redundancy involved in the choice of the length-scales $d$ and $l$. These were introduced in (A 2) and (A 11) as appropriate depth and radial length-scales, and we selected values $d \sim 100 \mathrm{~km}$ and $l \sim 200 \mathrm{~km}$ as illustrative of our expectations. In retrospect, we should have chosen these scales so that $r^{*}$ and $t^{*}$ are $O(1)$, and this we now attempt to do.

Consulting the model (3.8)-(3.10), we see that a change of the scale for $r$ has the single effect of changing the value of $\beta=\left(B_{n} / \omega^{3}\right)^{1 / n}$. Since our preliminary estimates of $d$ and $l$ give a value of $\beta \sim O(1)$, it makes sense to define $l$ so that $\beta=1$, and this we now do.

Define a 'viscous' length-scale

$$
l_{\eta}=\left[\frac{\kappa \exp (1 / \varepsilon)}{2 A \varepsilon^{2(n-1)}\left(\alpha T_{\mathrm{a}} \rho_{\mathrm{a}} g\right)^{n}}\right]^{1 /(n+2)}
$$

and a 'failure' length-scale

$$
l_{\tau}=\frac{\tau_{\mathrm{c}}}{\alpha T_{\mathrm{a}} \rho_{\mathrm{a}} g} .
$$

Equation (A 17) then gives

$$
R a=\frac{\nu^{2(n-1)} l^{n+2}}{l_{\eta}^{n+2}}
$$

so that (A 18) gives

$$
\omega^{3}=\frac{\nu^{2 n+3} l^{n+2} \varepsilon^{5}}{l_{\eta}^{n+2}},
$$

whence

$$
\beta=\left[\frac{B_{n} l_{\eta}^{n+2}}{\nu^{2 n+3} l^{n+2} \varepsilon^{5}}\right]^{1 / n} .
$$

From (A 59), we have

$$
d=\frac{l_{\tau}}{C}
$$

and thus we can choose $\beta=1$ by defining

$$
l=\left[\frac{\varepsilon^{5} d^{2 n+3}}{B_{n} l_{\eta}^{n+2}}\right]^{1 / n+1} .
$$

Specification of the second length-scale will be completed by a choice for $C$.

Finally, we define also a 'radiogenic' length-scale

$$
l_{Q}=\left(\frac{k T_{\mathrm{a}}}{\rho_{\mathrm{a}} H}\right)^{1 / 2},
$$

so that from (A 7)

$$
h=\left(\frac{d}{l_{Q}}\right)^{2} .
$$


The simplest choice for $C$ is $C=1$, but a more relevant choice is

$$
C=\frac{\left(1-T_{0}\right)^{2}}{6\left(T_{\mathrm{c}}-T_{0}\right)}
$$

for the following reason. When $h$ is small, $T$ relaxes (fairly rapidly, in fact) to the linear profile

$$
T=T_{0}+\left(1-T_{0}\right) \eta
$$

whence (4.4) becomes

$$
q \approx\left(\frac{1-T_{0}}{6 C}\right) \frac{1}{r^{1 / 2}} \int_{0}^{r} r^{1 / 2} s s_{r} \mathrm{~d} r
$$

and if $s, s_{r} \sim O(1)$, then $q \sim\left(1-T_{0}\right) / 6 C$. According to (4.10), failure occurs if $q / s \sim\left(T_{\mathrm{c}}-T_{0}\right) /\left(1-T_{0}\right)$, and equating the two orders of magnitude for $q$ leads to $(4.21)$.

For our standard set of parameters, we find $l_{\eta} \approx 16 \mathrm{~km}, l_{\tau} \approx 22 \mathrm{~km}$ (if $\tau_{\mathrm{c}}=$ $300 \mathrm{bar}$ ), and $l_{Q} \approx 600 \mathrm{~km}$. With $C$ given by (4.21), we have $C \approx 0.22$, and thus $d \approx 98 \mathrm{~km}$, which then implies $l \approx 180 \mathrm{~km}$. It is clear from the definitions of the parameters that variations in $n, T_{\mathrm{a}}$ and $\tau_{\mathrm{c}}$ can cause significant variation in these length-scales. However, our principal assumption, that $d<l$, is validated a posteriori by these estimates.

\section{(d) Numerical results}

By choosing $C$ via (4.21) and $\beta=1$, the lithosphere evolution problem depends on the parameters $n, T_{0}$ and $h$, as well as the initial lithosphere thickness $s_{0}$. We begin by taking fixed values $n=3.5, T_{0}=0.44, h=0.03$ (which we call the reference set), and compute the solution numerically until failure occurs, for various values of $s_{0}$.

Figure 6 shows a typical result of the profiles of $s$ and $q$ at the point of failure, while figure 7 shows the corresponding profile of $T_{q}$ as a function of $r$.

Figures 8 and 9 show the computed time of lithosphere failure $t^{*}$ and the radial distance $r^{*}$ of the failure zone from the plume centre, both plotted as (dimensionless) functions of the initial dimensionless lithosphere thickness $s_{0}$.

We can see from figures 8 and 9 that $t^{*}$ and $r^{*}$ are increasing functions of $s_{0}$, but are relatively constant for $0<s_{0}<1$. They take values

$$
r^{*}=44.7, \quad t^{*}=5.1,
$$

as $s_{0} \rightarrow 0$. We use these values below in assessing time and place of failure, since even if $s_{0}$ is larger, a similar result will be found for plumes which punch through the lithosphere (i.e. taking a boundary value $s_{0}<s_{00}$ ).

We note further that the numerical values of $r^{*}$ and $t^{*}$ are not as near to one as we had hoped. There is no inconsistency in this, since one could cosmetically re-choose $d$ and $l$ so that they were, but there is little point, as the dimensional results we identify below are not altered. The principal constraint on the analysis in this paper is that $d s\left(r^{*}, t^{*}\right) \ll l r^{*}$, and we see that for our standard parameter set, $d=98 \mathrm{~km}, s\left(r^{*}, t^{*}\right) \approx 3.7, l=180 \mathrm{~km}, r^{*} \approx 44.7$, whence $d s\left(r^{*}, t^{*}\right) \approx 363 \mathrm{~km}$ (lithosphere thickness at point of failure), and $l r^{*} \approx 6000 \mathrm{~km}$, so that the inequality is well satisfied. 


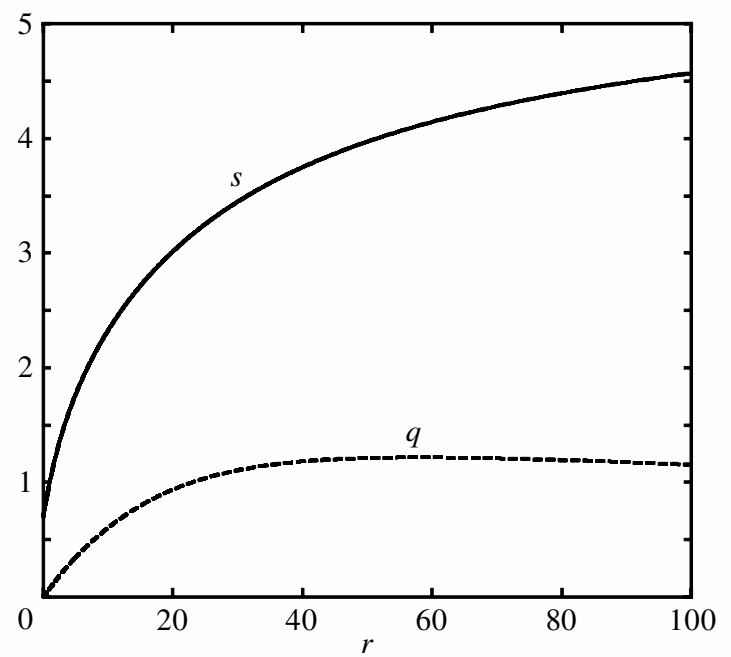

Figure 6. A typical situation at failure, where the plastic lid base $q$ and the lithosphere base $s$ are shown. Parameter values used are the reference set, and $s_{0}=0.7$.

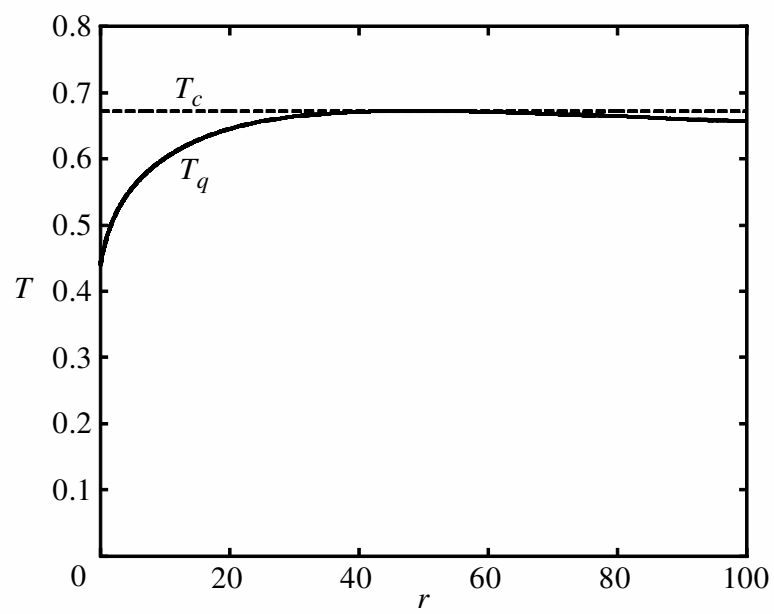

Figure 7. The profile of $T_{q}$ at failure, where it reaches the critical value $T_{\mathrm{c}}$.

Parameter values used are the reference set, and $s_{0}=0.7$.

The last point to note is that, from figure 7 , the temperature profile $T_{q}$ at the viscoplastic transition depth $z=q$ is a very flat function of $r$. Essentially, as $t$ approaches $t^{*}$, the lithosphere becomes fluid at all distances $r \gtrsim 20$. Thus, $r^{*}$ might be interpreted as an upper bound on the radial position of failure, and is a relatively imprecise estimate. As can be seen from figure 10, the viscosity is relatively constant at failure for $r \gtrsim r^{*} / 2$, and increases rapidly below this. In our estimates for failure location, we therefore use the value $r^{*} / 2$ rather than $r^{*}$.

\section{(e) Position and time of failure}

The predicted dimensional time and radial position of failure are given by $t_{\mathrm{f}}=$ $d^{2} t^{*} / \kappa$ and $r_{\mathrm{f}}=l r^{*} / 2$. Using the definitions of $d$ and $l$ in (4.17) and (4.18), we have, 


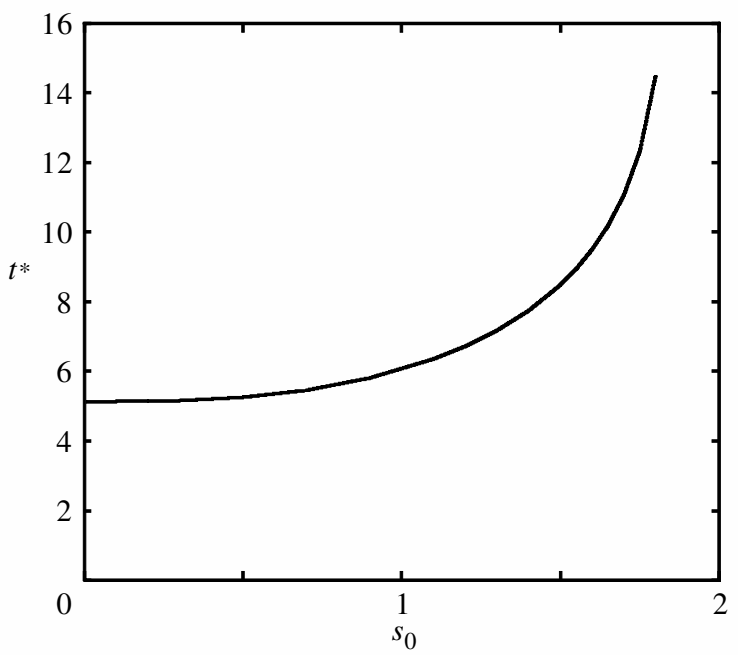

Figure 8. Time to failure $t^{*}$ as a function of the dimensionless initial lithosphere thickness $s_{0}$, standard parameter set.

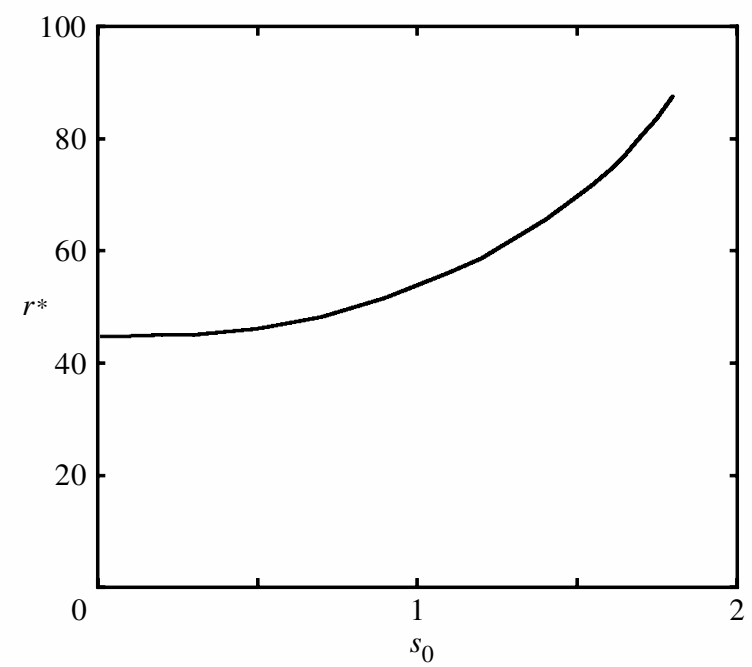

Figure 9. Failure location $r^{*}$ as a function of the dimensionless initial lithosphere thickness $s_{0}$, standard parameter set.

explicitly,

$$
t_{\mathrm{f}}=\frac{t^{*}}{\kappa\left(\alpha \rho_{\mathrm{a}} g C\right)^{2}}\left(\frac{\tau_{\mathrm{c}}^{2}}{T_{\mathrm{a}}^{2}}\right)
$$

and

$$
r_{\mathrm{f}}=\frac{r^{*}}{2}\left[\frac{2 A}{\kappa B_{n}\left(\alpha \rho_{\mathrm{a}} g\right)^{n+3}}\left(\frac{R}{E^{*} C}\right)^{2 n+3}\right]^{1 /(n+1)}\left[\tau_{\mathrm{c}}^{2 n+3} T_{\mathrm{a}}^{n} \exp \left\{-\frac{E^{*}}{R T_{\mathrm{a}}}\right\}\right]^{1 /(n+1)} .
$$

For our reference values, as given in table 1, we find

$$
r_{\mathrm{f}}=3004 \mathrm{~km}, \quad t_{\mathrm{f}}=1527 \mathrm{Ma} \quad \text { (reference set). }
$$




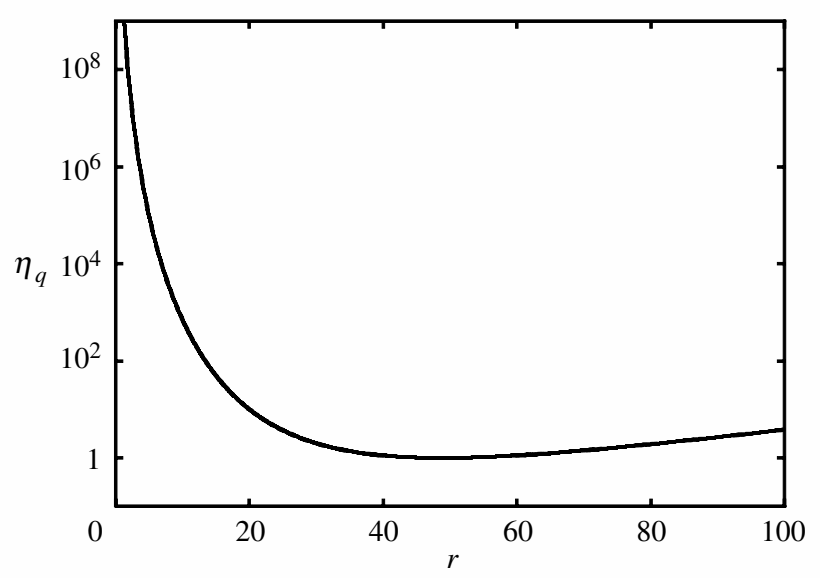

Figure 10. Variation of viscosity at $z=q$, at the point of failure. Same conditions as in figure 7.

Table 1. Assumed parameter values

(Those for $n, T_{\mathrm{a}}, \tau_{\mathrm{c}}, t^{*}$ and $r^{*}$ correspond to our reference set.)

\begin{tabular}{ccc}
\hline parameter & value & units \\
\hline$t^{*}$ & 5.1 & - \\
$\kappa$ & $10^{-6}$ & $\mathrm{~m}^{2} \mathrm{~s}^{-1}$ \\
$\alpha$ & $3 \times 10^{-5}$ & $\mathrm{~K}^{-1}$ \\
$\rho_{\mathrm{a}}$ & $3 \times 10^{3}$ & $\mathrm{~kg} \mathrm{~m}^{-3}$ \\
$g$ & 9 & $\mathrm{~m} \mathrm{~s}^{-2}$ \\
$C$ & 0.225 & - \\
$\tau_{\mathrm{c}}$ & 300 & $\mathrm{bar}\left(=10^{5} \mathrm{~Pa}\right)$ \\
$T_{\mathrm{a}}$ & 1700 & $\mathrm{~K}$ \\
$r^{*}$ & 44.7 & - \\
$A$ & $3 \times 10^{4}$ & $\mathrm{MPa}^{-n} \mathrm{~s}^{-1}$ \\
$R$ & 8.3 & $\mathrm{~J} \mathrm{~mol}^{-1} \mathrm{~K}^{-1}$ \\
$E^{*}$ & 535 & $\mathrm{~kJ} \mathrm{~mol}^{-1}$ \\
$n$ & 3.5 & - \\
$B_{n}$ & $1.98 \times 10^{-5}$ & - \\
\hline
\end{tabular}

Now we wish to explore the sensitivity of the values of $t_{\mathrm{f}}$ and $r_{\mathrm{f}}$ to variations in some of the parameters. These values are likely to be most sensitively dependent on the asthenospheric temperature $T_{\mathrm{a}}$, the flow-law exponent $n$ and the failure stress $\tau_{\mathrm{c}}$, none of which can be presumed to be adequately known for Venus. Figures 11 and 12 show the variation of $t_{\mathrm{f}}$ and $r_{\mathrm{f}}$ when each of these is varied in turn. In so doing, we fix $T_{0}\left(=T_{\mathrm{s}} / T_{\mathrm{a}}\right)$, which will not vary much over the small range of $T_{\mathrm{a}}$ we consider. We also fix $h$, partly on the basis that, if it is small, its precise value should not affect the solution significantly. Hence, $r^{*}, t^{*}$ and $C$ will depend only on $n$, and this dependence is shown in table 2 .

In figure 11, we see that failure occurs at times in the range 200-800 Ma for failure stresses in the range 100-200 bar (10-20 MPa), which does not seem unreasonable. The radial position of failure is in the range $200-1200 \mathrm{~km}$ for an asthenospheric temperature of $1700 \mathrm{~K}$, or the lower range $80-360 \mathrm{~km}$ for the more Earth-like astheno- 

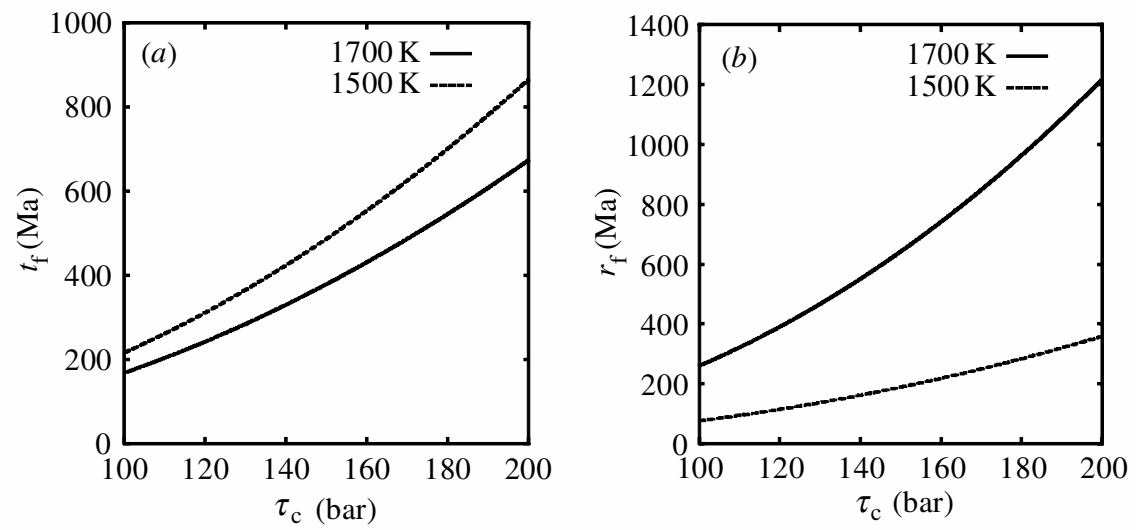

Figure 11. Variation of (a) failure time $t_{\mathrm{f}}$ as given by (4.25), and (b) failure radius $r_{\mathrm{f}}$ as given by (4.26), as a function of $\tau_{\mathrm{c}}$ for values $T_{\mathrm{a}}=1700 \mathrm{~K}$ and $1500 \mathrm{~K}$. Other parameters are standard, in particular $n=3.5$.
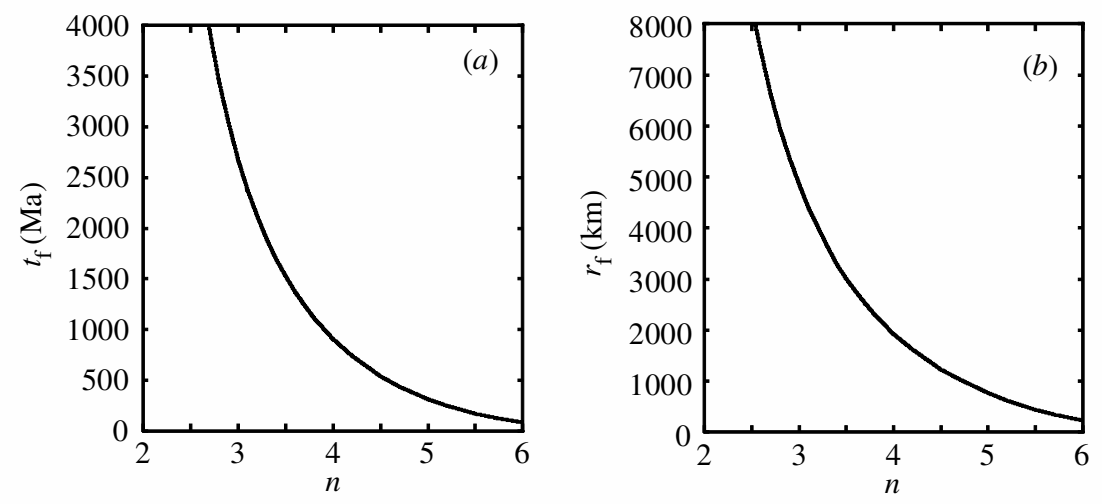

Figure 12. Variation of $(a)$ failure time $t_{\mathrm{f}}$ as given by (4.25), and $(b)$ failure radius $r_{\mathrm{f}}$ as given by (4.26), as a function of $n$. Other parameters as standard, in particular $T_{\mathrm{a}}=1700 \mathrm{~K}$ and $\tau_{\mathrm{c}}=300$ bar $(30 \mathrm{MPa})$.

Table 2. Variation of parameters with $n$

\begin{tabular}{llccc}
\hline$n$ & $C$ & $B_{n}$ & $t^{*}$ & $r^{*}$ \\
\hline 2.5 & 0.157 & $8.68 \times 10^{-4}$ & 8.8 & 74.0 \\
3 & 0.187 & $1.39 \times 10^{-4}$ & 6.2 & 55.5 \\
3.5 & 0.225 & $1.98 \times 10^{-5}$ & 5.1 & 44.8 \\
4 & 0.274 & $2.53 \times 10^{-6}$ & 4.5 & 38.4 \\
5 & 0.429 & $3.15 \times 10^{-8}$ & 3.8 & 31.3 \\
\hline
\end{tabular}

spheric temperature of $1500 \mathrm{~K}$. The variation of $t_{\mathrm{f}}$ and $r_{\mathrm{f}}$ with the flow exponent is shown in figure 12. The decrease in both with increasing $n$ is largely due to the variation of $l$ and $d$ with $n$ (both $t^{*}$ and $r^{*}$ decrease by a factor of two between $n=3$ and $n=6$ ). This figure exemplifies the crucial importance of the nonlinearity of the flow law in facilitating failure in geologically sensible times. 


\section{Discussion}

We have set out in this paper to examine whether the hypothesis of episodic subduction on Venus advanced by Turcotte (1993) is quantitatively viable. Our method for assessing the hypothesis is to advance a mathematical model of mantle convection, in whose framework the hypothesis can be evaluated. Specifically, we have focused on the idea, advanced by Sandwell \& Schubert (1992), that trenches surrounding certain coronae have the signature of subduction zones on the Earth. The inevitable result of this process is that, given the particular model we choose, we obtain parametric constraints on the model, within which the hypothesis appears feasible.

The mathematical model represents convection in a planetary mantle, using a fairly realistic rheology. Our principal hypothesis is that mantle rocks will fail by effectively plastic creep if subjected to sufficiently high shear stresses for sufficiently long times, and that these stresses are naturally generated in the high-Rayleighnumber convection of a strongly variable viscosity system. This latter statement is true (Fowler 1985), so that the efficacy of our model relies on the way in which we model the rheology. In our previous work (Fowler 1993; Fowler \& O'Brien 1996) we used a purely viscoplastic rheology, which is somewhat at odds with the sort of viscoelastic rheology that is commonly used. Let us therefore rehearse our discussion of this point.

Viscous creep in polycrystalline rocks can occur in laboratory experiments (Goetze 1978) at very high deviatoric stresses, without apparent failure. The problem is that such results must be extrapolated through many orders of magnitude to mantle conditions. It is well known that such deformational experiments often describe secondary creep, but that grain recrystallization leads to tertiary accelerating creep over time-scales which are inaccessible in the laboratory. Therefore, it is plausible to suppose that in fact such 'superplastic' creep may be truly modelled by a plastic yield stress such as is used in the study of metal fatigue (Hobbs \& Ord 1988). Furthermore, we believe that the Earth itself provides a laboratory in which this plastic yield occurs; three locations where deformation may be effectively described by viscoplastic creep are: the Benioff zones in subducting lithosphere; lithospheric fracture zones, for example, in oceanic plates; and continental collisions, such as that between India and Asia. The buoyant stress induced across a $100 \mathrm{~km}$ thick slab of lithosphere with a temperature deficit of $1000 \mathrm{~K}$ is of the order of 300 bar (30 MPa). Over geological time, such stresses cannot be maintained by steady creep processes, and slip events occur. The simplest model of such stick-slip motion is via an effective coefficient of friction, or in other words a yield stress. Therefore, we consider that the plastic yield model is viable, and moreover that reasonable values for $\tau_{\mathrm{c}}$ on the Earth are of the order of 300 bar (30 MPa).

In our discussion of yield, we have considered the classical concept of brittleductile yield in a viscoelastic lithosphere (figure 4). The nomenclature is unhelpful, since, although the brittle yield of Byerlee's law would indeed be modelled by an elastic-plastic rheology, with Byerlee's law corresponding to a slice of the yield surface (Hill 1950), the 'ductile' yield curve is not a yield curve at all, but simply a statement of the stress required to deform mantle rock at a certain strain rate. In fact, we have shown that the classical Maxwell model of viscoelasticity also allows for elastic-plastic modelling, providing that the 'viscosity' becomes unconstrained on the (e.g. Tresca) yield surface. 
In summary, our rheological model is of a viscoelastic (Maxwell) material with stress and temperature-dependent rheology, together with a plastic yield stress. This latter concept is the only departure from convention, and our primary basis for proposing it is the existence of such an effective yield stress in terrestrial plate tectonics. Pressure dependence through an activation volume is not included, but for the sorts of values usually quoted $\left(17 \mathrm{~cm}^{3} \mathrm{~mol}^{-1}\right)$, the effect is small near the lithosphere where our analysis focuses.

Given this rheology, the remainder of the paper consists of finding the solution which describes the convective outflow associated with a mantle plume. The solution is derived using a sequence of asymptotic approximations, each of which is parametrically controlled, without any arbitrary assumptions whatsoever. Although the method is necessarily convoluted, the logical progression is based on previous work (Morris \& Canright 1984; Fowler 1985, 1993; Reese et al. 1998) in simpler systems where perhaps the analysis is less opaque. In that sense, the conclusions follow rigorously from the yield hypothesis. There is some leeway insofar as one can never be certain exactly how extreme the conditions must be before the results are 'accurate'; experience shows that generally in such circumstances, results are qualitatively accurate, and quantitatively reasonable.

We consider a situation designed to represent conditions in a Venusian mantle following an overturning (roll-over) event. In this event, the cold lithosphere sinks to the base of the mantle, and rests there while a hot thermal boundary layer grows underneath it. The initial temperature above this basal region is uniform (at $T_{\mathrm{a}}$ ) but after a while the lower boundary layer becomes unstable, as envisaged by Howard (1966), and it sends up thermal plumes, which we suppose build coronae at the surface. During the hiatus, the top surface starts to cool, and a cold sticky lithosphere grows downward into the mantle. It is gravitationally unstable, but extremely viscous, which precludes its subduction under normal planetary mantle conditions.

Now if a plume impacts on this growing lithosphere, a sub-lithospheric radial outflow will occur, similar to that which occurs in steady two-dimensional convection (Fowler 1985). The structure of this flow is well known: the lithosphere has exponentially small (in the viscosity number $\varepsilon=R T_{\mathrm{a}} / E^{*}$ ) velocity, high stresses, and is joined to the underlying mantle by a thin delamination layer, in which the temperature gradient jumps from conductive to isothermal, and, importantly, the scales of which are self-determining.

Above the delamination layer, the stresses increase dramatically in the lithosphere, and any reasonable value of the yield stress will cause a zone of failure adjoining the surface. Indeed, we suppose that the common observation of bent and crumpled surface rocks is an indication of just such failure. In dimensionless units, we denote the depth to the delamination layer as $s(r, t)$, and the depth of the plastic failure zone as $q(r, t)$, and our aim is then to derive evolution equations for these. If the viscosity at $q$ becomes comparable to that of the underlying mantle at $r=r_{\mathrm{c}}$, then at $r_{\mathrm{c}}$ the effective viscosity of the entire lithospheric column becomes equal to that of the underlying mantle, and we suppose that subduction will be initiated. We have no way of knowing in advance whether this will occur, or where.

If subduction is initiated in this way, then, as soon as the heavy lithosphere begins to plunge into the mantle, the negative buoyancy associated with it increases dramatically. The descending slab will drive a suddenly vigorous convective flow towards the subduction site, and at the same time the subduction trench will migrate backwards 
away from the plume head; as it does so, the lithosphere will peel away from the mantle and collapse through the mantle. One resultant possibility is an entire resurfacing of the planet, as the old lithosphere sinks towards the core-mantle boundary. This could only be prevented if sufficient upwelling material becomes available to replace the sinking mantle, or if the subducting mantle is jammed in its descent, for example, by a pressure or chemically induced increase in viscosity. Planetary resurfacing would seem to be a likely consequence of an initial failure event.

The analysis used to calculate for $s$ and $q$ follows the path found by Fowler (1985), who computed the lithosphere stresses and $s$ in steady, purely viscous, twodimensional flow; this was generalized to steady two-dimensional flow with a plastic failure zone (and a viscoplastic rheology) by Fowler (1993), and to an unsteady twodimensional viscoplastic flow by Fowler \& O'Brien (1996). In the present paper, we have treated the unsteady flow problem in a cylindrical geometry, and, exhorted by the reviewers of an earlier draft, we have allowed the rheology to be fully viscoelasticplastic, with a stress dependent nonlinear rheology, and we have included the quantitative effect of radiogenic heating. The stress dependence and internal heating make for minor changes in method, and we have been able to sidestep the complications associated with the elastic part of the rheology, firstly because lithospheric strains will be so small that material derivatives are not required in the constitutive law, and secondly because the elevated surface temperature of Venus turns out to be enough to warrant an assumption of no elastic layer between the upper elastic-plastic region and the viscous region below. Consequently, the upper region is in fact viscoplastic, and our previous analyses carry through as before; this would not apply to the Earth, however.

Following through the analysis, we derive a reduced model for the determination of $s$ and $q$ : this is (3.8)-(3.10). It is only in this final phase of the analysis that the influence of the plume is manifested by the boundary condition (4.2). The single feature of our solution procedure which is artificial is the assumption of a prescribed lithosphere depth at the upwelling, rather than a formula derived from detailed consideration of plume dynamics. We do not consider this to be a serious dereliction.

The results of the analysis, convoluted though they may be, are clear, and are detailed in the conclusions. The onset of subduction following a global tectonic rollover is a likely possibility, and the time- and space-scales for its occurrence are consistent with observations on Venus and with plausible parameter values of the material constants.

\section{Conclusions}

Common discussions of brittle-ductile yield, as exemplified for viscoelastic materials in figure 4, are misleading, as the viscous 'yield' is nothing other than permanent creep. Nevertheless, plastic yield has meaning in mantle minerals, both in the accepted form of Byerlee's law, which may represent the brittle failure of elastic material, and in the viscous mantle, where it is manifested in the Earth through the existence of deep earthquakes, fracture zones, and continental collisions, which are evidences of behaviour that may effectively be modelled in the long term by a viscoplastic rheology of Tresca or Von Mises type. Direct inferences of the corresponding yield stress give values of $c a .300$ bar $(30 \mathrm{MPa})$ on the Earth.

Proc. R. Soc. Lond. A (2003) 
Following a supposed mantle roll-over event on Venus $500 \mathrm{Myr}$ BP, convective plumes will emerge from the core-mantle boundary, and it seems likely that these would appear before the establishment of a general cellular circulation. The impingement of a plume at the growing lithosphere causes uplift of the surface and lithosphere thinning. The resultant radial outflow causes thinning of the lithosphere away from the plume, and the resultant stresses induced by the large buoyancy cause the growth of a plastically failed zone at the surface, in which the effective viscosity is much reduced. At a time $t_{\mathrm{f}}$ and radial distance $r_{\mathrm{f}}$ the effective viscosity of the whole lithosphere becomes equal to that of the underlying mantle, and the lithosphere becomes fluid. For a yield stress $\tau_{\mathrm{c}}$ of $200 \mathrm{bar}(20 \mathrm{MPa})$, flow-law exponent $n=3.5$, and asthenospheric temperature $T_{\mathrm{a}}=1500 \mathrm{~K}$, the failure time is $c a .800 \mathrm{Myr}$, and the failure distance is $c a .400 \mathrm{~km}$. These values vary for different choices of $\tau_{\mathrm{c}}, n$ and $T_{\mathrm{a}} ; t_{\mathrm{f}}$ decreases a little as $T_{\mathrm{a}}$ increases, but $r_{\mathrm{f}}$ increases dramatically. Both values increase with increasing $\tau_{\mathrm{c}}$ and decreasing $n$. These values suggest that Turcotte's (1993) scenario of episodic overturn with a time-scale of the order of $500 \mathrm{Myr}$, mediated by subduction-like failure zones at the rim of coronae, is consistent with theoretical models of mantle convection, providing a failure stress of order 200 bar exists.

A prominent feature of the analysis is that, when lithospheric failure occurs, it does so in a way which renders all the lithosphere outside the failure-radius fluid, while the viscosity jumps rapidly inside this radius. The picture of plume emplacement that this suggests is the following. A massive thermal plume erupts from the core-mantle boundary following overturn, and the resultant deposition of cold surface rock there. This plume rises rapidly towards the thin, rigid, young lithosphere, which it causes to rise, like a fist pushing up under a stretched sheet. This broad topographic rise is maintained until failure, when, suddenly, the lithosphere outside the plume area collapses viscously, while the plume maintains its role as a rigid indenter under a fluid blanket. As a consequence, the surrounding, now fluid, lithosphere will collapse, and one would expect to see the sort of transient trenches that are observed on some coronal rims. A striking numerical illustration of this scheme of events is given by Smrekar \& Stofan (1997). They found that a plume incident on the lithosphere spreads under it and causes a delamination of the lithosphere, and a sinking cold plume, which migrates back towards the plume head. As they say, this scenario resembles that of coronae on Venus which exhibit the arcuate trenches, except that in their model they would need some kind of lithosphere weakness in order to predict the trenches. In this paper we have presented such a weakening mechanism.

Whether or not this weakening actually signifies incipient subduction is less clear: it is certainly a realistic possibility. But even if not, what we are led to suggest is that the maintenance of the coronal elevation and the presence of a surrounding depression are consistent with the formation of a sudden fluidization failure of the surrounding lithosphere. One would then expect subduction to occur elsewhere, as the cold but fluid lithosphere sinks into the mantle. Perhaps this is what is indicated by the chasmata (Jurdy \& Stefanick 1999).

It is always attractive to confirm the results of analytic solutions with direct numerical computations. A difficulty in doing this in planetary mantle convection arises through the issue of extrapolation. A quantity of relevance in variable viscosity convection is the viscosity contrast $\Delta \eta$, which we can define in the present case as

$$
\Delta \eta=\exp \left[\frac{E^{*}}{R}\left(\frac{1}{T_{\mathrm{s}}}-\frac{1}{T_{\mathrm{a}}}\right)\right] \approx \exp \left[\frac{E^{*}}{R T_{\mathrm{a}}}\right],
$$


if $T_{\mathrm{a}}=1500 \mathrm{~K}$ and $T_{\mathrm{s}}=750 \mathrm{~K}$. We see from (4.26) that, approximately,

$$
r_{\mathrm{f}} \propto \Delta \eta^{-1 /(n+1)}
$$

Using the values in table 1 (but $T_{\mathrm{a}}=1500 \mathrm{~K}$ ), we have

$$
\Delta \eta \approx 4.6 \times 10^{18}
$$

The best numerical computations can reach a $\Delta \eta$ value of about $10^{10}$. The corresponding position of failure for this lower value of the viscosity contrast would be a factor $\left(4.6 \times 10^{8}\right)^{1 /(n+1)}$ greater; for $n=3.5$, this is a factor of 84 . For failure at $400 \mathrm{~km}$ with $\Delta \eta \sim 10^{18}$, we would have $r_{\mathrm{f}} \approx 33000 \mathrm{~km}$ for $\Delta \eta \sim 10^{10}$. Failure would not be seen.

We thank Don Turcotte for illuminating discussions, and Philip England for helpful advice.

\section{Appendix A.}

\section{(a) Scaling the equations}

It is convenient to follow the recipe given by Fowler \& O'Brien (1996), though the presence of the flow-law exponent $n$ causes some slight (but important) modification. Extension of the boundary layer theory, for the case of Cartesian two-dimensional flow, to values of the flow-law exponent $n>1$ has been done by Reese et al. (1998, 1999), and much of the development below parallels that work.

We subtract the lithostatic pressure $\rho_{\mathrm{a}} g z$ from the pressure, and then scale the stresses as

$$
p-\rho_{\mathrm{a}} g z, \tau_{r r}, \tau_{r z}, \tau_{z z} \sim \frac{\eta_{\mathrm{a}} \kappa}{l^{2}}
$$

where $\eta_{\mathrm{a}}$ is a viscosity scale, to be defined below, and $l$ is a convenient length-scale; since we hope to find failure occurring at distances of the order of several hundred kilometres from the centre of a plume, this gives a natural choice for the value of $l$, although we will in fact eventually prescribe it in terms of other parameters of the problem. In addition we choose scales for the other variables

$$
T \sim T_{\mathrm{a}}, \quad \eta \sim \eta_{\mathrm{a}}, \quad t \sim \frac{l^{2}}{\kappa}, \quad \boldsymbol{x} \sim l, \quad \boldsymbol{u} \sim \frac{\kappa}{l} .
$$

The resulting dimensionless equations are then

$$
\left.\begin{array}{c}
\frac{1}{r} \frac{\partial}{\partial r}(r u)+\frac{\partial w}{\partial z}=0, \\
\frac{\partial p}{\partial r}=\frac{1}{r} \frac{\partial}{\partial r}\left(r \tau_{r r}\right)+\frac{\partial \tau_{r z}}{\partial z}+\frac{\tau_{r r}+\tau_{z z}}{r}, \\
\frac{\partial p}{\partial z}=\frac{1}{r} \frac{\partial}{\partial r}\left(r \tau_{r z}\right)+\frac{\partial \tau_{z z}}{\partial z}+R a^{*}(1-T),
\end{array}\right\}
$$




$$
\left.\begin{array}{c}
\tau_{r r}=2 \eta\left[\frac{\partial u}{\partial r}-\delta\left(\frac{\partial \tau_{r r}}{\partial t}-c \frac{\partial p}{\partial t}\right)\right], \\
\tau_{z z}=2 \eta\left[\frac{\partial w}{\partial z}-\delta\left(\frac{\partial \tau_{z z}}{\partial t}-c \frac{\partial p}{\partial t}\right)\right], \\
\tau_{r z}=\eta\left[\frac{\partial u}{\partial z}+\frac{\partial w}{\partial r}-\delta \frac{\partial \tau_{r z}}{\partial t}\right], \\
\frac{\partial T}{\partial t}+u \frac{\partial T}{\partial r}+w \frac{\partial T}{\partial z}=\nabla^{2} T+h^{*}, \\
\tau^{2}=\tau_{r z}^{2}+\frac{1}{2}\left[\tau_{r r}^{2}+\tau_{z z}^{2}+\left(\tau_{r r}+\tau_{z z}\right)^{2}\right] .
\end{array}\right\}
$$

(A 3 cont.)

The dimensionless parameters are a form of Rayleigh number

$$
R a^{*}=\frac{\alpha \rho_{\mathrm{a}} g T_{\mathrm{a}} l^{3}}{\eta_{\mathrm{a}} \kappa},
$$

a viscosity number

$$
\varepsilon=\frac{R T_{\mathrm{a}}}{E^{*}}
$$

a viscoelastic (Deborah) number

$$
\delta=\frac{\eta_{\mathrm{a}} \kappa}{2 \mu l^{2}}
$$

and an internal heating number

$$
h^{*}=\frac{\rho_{\mathrm{a}} l^{2} H}{k T_{\mathrm{a}}},
$$

where we define the volumetric heating $Q$ in terms of the internal heating per unit mass, $H$, as $Q=H / c_{p}$. Since $E=\mu(3 \lambda+2 \mu) /(\lambda+\mu)$ and $\sigma=\lambda /[2(\lambda+\mu)]$, where $\sigma$ is Poisson's ratio, the elastic parameter $c$ is given by

$$
c=\frac{1-2 \sigma}{3(1+\sigma)} \text {. }
$$

The rheological parameter $\Lambda$ is defined by

$$
\Lambda=\frac{1}{2 A \eta_{\mathrm{a}}}\left(\frac{l^{2}}{\eta_{\mathrm{a}} \kappa}\right)^{n-1} \exp \left(\frac{E^{*}}{R T_{\mathrm{a}}}\right),
$$

and will be fixed below by a suitable choice of $\eta_{\mathrm{a}}$ (which depends on the size of the stresses in the asthenosphere).

In due course we will define an appropriate actual Rayleigh number, $R a$, for the flow. Typical values of $R a$ and $\varepsilon$ are likely to be large and small, respectively. It is on this basis that an approximate boundary-layer analysis can be attempted. In fact, such an analysis shows that the dynamics of the stagnant lid is more or less selfdetermining, and is largely independent of the details of any convective flow below, 


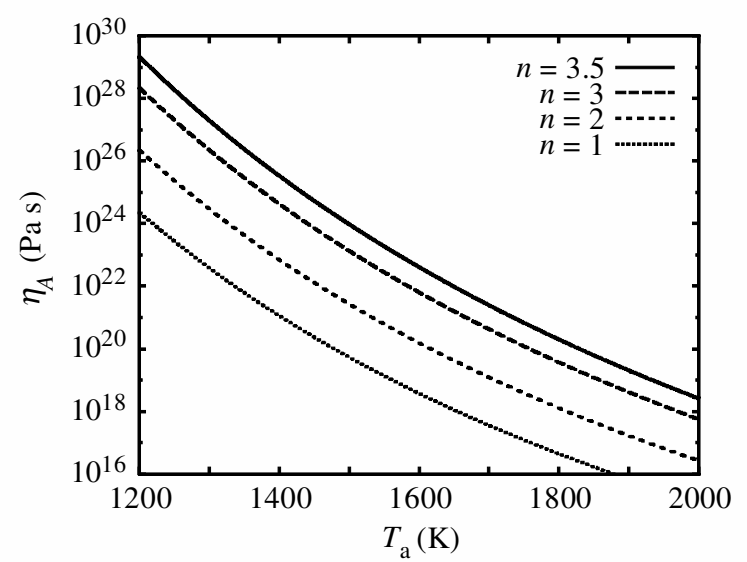

Figure 13. Asthenospheric viscosity scale $\eta_{A}$ (Pa s) as a function of asthenospheric temperature $(\mathrm{K})$, for various values of $n$. The implications of these curves are that vigorous sub-lithospheric convection $\left(\eta_{A}<10^{22} \mathrm{Pas}\right)$ on Venus requires asthenospheric temperatures of at least $1700 \mathrm{~K}$, if $n=3.5$. Note that this graph is not of viscosity varying with temperature at different depths in the mantle.

providing the lid is of a thickness less than $l$. The basis for this latter supposition is that the thermal time-scale $l^{2} / \kappa$ is $c a .3000 \mathrm{Myr}$, whereas we are interested in a timescale an order of magnitude smaller. If we define $\nu^{2}$ to be the ratio of this shorter time-scale to $l^{2} / \kappa$, and presume that $\nu^{2} \ll 1$, then it is appropriate to rescale the variables in the following way, in order that the dimensionless variables be $O(1)$; this leads to what we call the slab scales (slab refers to the cold lid, or lithosphere):

$$
\left.\begin{array}{c}
z \sim \nu, \quad u \sim R a^{* 3 / 5} \nu, \quad w \sim R a^{* 3 / 5} \nu^{2}, \quad t \sim \nu^{2}, \quad \eta \sim\left(\nu \varepsilon R a^{* 1 / 5}\right)^{2}, \\
\tau \sim \tau_{r z} \sim \nu^{2} R a^{*}, \quad p \sim \nu R a^{*}, \quad \tau_{r r} \sim \tau_{z z} \sim \nu^{3} R a^{*} .
\end{array}\right\}
$$

This abstruse choice of scales follows directly from our previous work (Fowler 1985, 1993; Fowler \& O'Brien 1996), and is motivated by the need to balance advection and conduction in the delamination layer below (see (A 29)), as well as to equate shear stress gradient with buoyancy. By choosing such a balance, we are able to effect a transition between the stress and temperature in the slab and the same quantities in the deep mantle. The particular choice of slab scales then arises as that appropriate for matching to the delamination layer.

Note that the quantity

$$
d=\nu l
$$

is the relevant depth-scale for the lithosphere, and, like $l$, it will eventually be prescribed in terms of physical parameters of the problem. In the meantime, we formally assume that $d \ll l$. The rescaled continuity equation allows a Stokes stream function $\psi$ via

$$
u=\frac{1}{r} \frac{\partial \psi}{\partial z}, \quad w=-\frac{1}{r} \frac{\partial \psi}{\partial r},
$$


and then the other rescaled equations are

$$
\begin{gathered}
\frac{\partial p}{\partial r}=\frac{\partial \tau_{r z}}{\partial z}+\nu^{2}\left[\frac{1}{r} \frac{\partial}{\partial r}\left(r \tau_{r r}\right)+\frac{\tau_{r r}+\tau_{z z}}{r}\right], \\
\frac{\partial p}{\partial z}=1-T+\nu^{2}\left[\frac{1}{r} \frac{\partial}{\partial r}\left(r \tau_{r z}\right)+\frac{\partial \tau_{z z}}{\partial z}\right] \\
\frac{1}{\varepsilon^{2}} \tau_{r z}=\eta\left[\frac{1}{r} \frac{\partial^{2} \psi}{\partial z^{2}}-\nu^{2} \frac{\partial}{\partial r}\left(\frac{1}{r} \frac{\partial \psi}{\partial r}\right)-\bar{\delta} \frac{\partial \tau_{r z}}{\partial t}\right], \\
\frac{1}{\varepsilon^{2}} \tau_{r r}=2 \eta\left[\frac{\partial}{\partial r}\left(\frac{1}{r} \frac{\partial \psi}{\partial z}\right)-\bar{\delta}\left(\frac{\partial \tau_{r r}}{\partial t}-\bar{c} \frac{\partial p}{\partial t}\right)\right], \\
\frac{1}{\varepsilon^{2}} \tau_{z z}=2 \eta\left[-\frac{1}{r} \frac{\partial^{2} \psi}{\partial z \partial r}-\bar{\delta}\left(\frac{\partial \tau_{z z}}{\partial t}-\bar{c} \frac{\partial p}{\partial t}\right)\right] \\
\eta=\left(\frac{\varepsilon^{2}}{\tau}\right)^{n-1} \exp \left(-\frac{\theta}{\varepsilon}\right), \theta=1-\frac{1}{T}, \\
\frac{\omega^{3}}{\partial t}\left[\frac{\partial \psi}{\varepsilon^{3} r} \frac{\partial T}{\partial r}-\frac{\partial \psi}{\partial r} \frac{\partial T}{\partial z}\right]=\frac{\partial^{2} T}{\partial z^{2}}+\nu^{2}\left[\frac{\partial^{2} T}{\partial r^{2}}+\frac{1}{r} \frac{\partial T}{\partial r}\right]+h, \\
\tau^{2}=\tau_{r z}^{2}+\frac{1}{2} \nu^{2}\left\{\tau_{r r}^{2}+\tau_{z z}^{2}+\left(\tau_{r r}+\tau_{z z}\right)^{2}\right\}
\end{gathered}
$$

where the modified parameters are defined by

$$
\bar{\delta}=\delta R a^{* 2 / 5}, \quad \bar{c}=c / \nu^{2}, \quad \omega=\nu \varepsilon R a^{* 1 / 5}, \quad h=\nu^{2} h^{*},
$$

and we now choose to define

$$
\Lambda=R a^{* 3(n-1) / 5} \omega^{2 n} .
$$

Bearing in mind the rescaling in (A 10), and anticipating (see (A 28)) that $\tau \sim \varepsilon^{2}$ in the asthenosphere, we find that the asthenospheric viscosity scale $\eta_{A}=\eta_{\mathrm{a}} \omega^{2}$ is given by

$$
\eta_{A}=\left[\frac{E^{* 2}}{\alpha \rho_{\mathrm{a}} g l \nu^{2} R^{2} T_{\mathrm{a}}^{3}}\right]^{n-1} \frac{\exp \left(E^{*} / R T_{\mathrm{a}}\right)}{2 A} .
$$

(In passing, we note that this gives explicit support to Tozer's (1972) concept of a viscously self-regulating mantle.) Figure 13 shows how this asthenospheric viscosity scale varies with the ambient temperature. It is clear that Venusian asthenospheric temperatures must be hotter than those on the Earth if the viscosity is going to approach the sort of low values which we might expect. With a thick, stagnant lithosphere, this is not in itself surprising. We can now finally define the relevant Rayleigh number for the flow. This is

$$
R a=\frac{\alpha \rho_{\mathrm{a}} g T_{\mathrm{a}} l^{3}}{\eta_{A} \kappa},
$$

and it follows from (A 14) that

$$
\omega=(\nu \varepsilon)^{5 / 3} R a^{1 / 3} .
$$


At this point it is useful to get some idea of the size of the parameters. If we take $\alpha=3 \times 10^{-5} \mathrm{~K}^{-1}, \rho_{\mathrm{a}}=3 \times 10^{3} \mathrm{~kg} \mathrm{~m}^{-3}, g=9 \mathrm{~m} \mathrm{~s}^{-2}, c_{p} \approx 10^{3} \mathrm{~J} \mathrm{~kg}^{-1} \mathrm{~K}^{-1}, T_{\mathrm{a}}=$ $1700 \mathrm{~K}, l=300 \mathrm{~km}, \kappa=10^{-6} \mathrm{~m}^{2} \mathrm{~s}^{-1}$, and presume a value of $\eta_{A}=3 \times 10^{21} \mathrm{Pas}$, then

$$
R a \approx 10^{4}
$$

If $E^{*}=535 \mathrm{~kJ} \mathrm{~mol}^{-1}, A=3 \times 10^{4} \mathrm{MPa}^{-n} \mathrm{~s}^{-1}, n=3.5$ (Kirby \& Kronenberg 1987), and $R=8.3 \mathrm{~J} \mathrm{~mol}^{-1} \mathrm{~K}^{-1}$, then $E^{*} / R \approx 64000 \mathrm{~K}$ and thus

$$
\varepsilon \approx 0.027 \text {. }
$$

If $\nu \approx 0.3$, then $(\mathrm{A} 18)$ implies

$$
\omega \approx 0.007 \text {. }
$$

Then $\eta_{\mathrm{a}} \approx 0.6 \times 10^{25} \mathrm{Pas}$ and $R a^{*} \approx 0.5$, so that if $\mu=0.6 \times 10^{11} \mathrm{~Pa}$, then the modified viscoelastic parameter $\delta$ in (A 14) is

$$
\bar{\delta} \approx 0.4 \times 10^{-2},
$$

while, with $H=5 \times 10^{-12} \mathrm{~W} \mathrm{~kg}^{-1}, k=3 \mathrm{~W} \mathrm{~m}^{-1} \mathrm{~K}^{-1}$, the heating parameter

$$
h \approx 0.033 \text {. }
$$

Finally,

$$
\bar{c} \approx 1.3
$$

assuming Poisson's ratio is $\sigma=0.25$.

The boundary conditions are those of no stress and prescribed temperature at the top surface. If the dimensional surface temperature is $T_{\mathrm{s}}$, then

$$
\psi=0, \quad T=T_{0}, \quad \tau_{r z}=0, \quad p-\nu^{2} \tau_{z z}=\Delta_{\mathrm{s}} \quad \text { at } z=0,
$$

where $T_{0}=T_{\mathrm{s}} / T_{\mathrm{a}}$, and the uplift at the surface is defined as

$$
h_{\mathrm{s}}=\alpha T_{\mathrm{a}} \nu l \Delta_{\mathrm{s}},
$$

and is assumed small (in the sense that $\alpha T_{\mathrm{a}} \Delta_{\mathrm{s}} \ll 1$ ) so that the surface conditions can be linearized about $z=0$. The conditions at large $z$ are discussed further below (see (A 30) and (A 31)).

We now proceed to analyse the equations, as in Fowler \& O'Brien (1996), in the separate regions of the flow. We recall that these regions are a plastic lid $0<z<q$, where plastic flow occurs but flow is small, a viscous lid $q<z<s$, and a delamination layer near $z=s$, which joins the stagnant lid to the mobile convection below. The assumption that there is no elastic core relies on the fact that the elastic terms proportional to $\bar{\delta}$ remain negligible.

\section{(b) Lithosphere}

In the lithosphere, i.e. the stagnant lid $z<s$ (see figure 5), we anticipate that $\psi$ is exponentially small (as $\eta$ is exponentially large), and thus the temperature satisfies

$$
\left.\begin{array}{rl}
T_{t} & =T_{z z}+h, \\
T & =T_{0} \quad \text { on } z=0, \\
T & =1, \quad T_{z}=\Gamma \quad \text { on } z=s .
\end{array}\right\}
$$

Here $T=1$ at $z=s$ in order to match to the asthenospheric temperature $(T=1)$ below $z=s$, while the temperature gradient $\Gamma$ is as yet unspecified, but is determined by matching through the shear layer at $z=s$. 


\section{(c) Delamination layer}

This is a shear layer at the base of the lithosphere (see figure 5), which adjusts the temperature to its asthenospheric value, and allows the stream function to begin to adjust towards the convective velocity field below. Importantly, it is essentially self-determining. We put

$$
\left.\begin{array}{c}
z=s+\varepsilon \zeta, \quad T=1+\varepsilon \phi, \\
\tau_{p q}=\varepsilon^{2} T_{p q}, \quad \psi=\varepsilon^{2} \Psi, \quad p=\varepsilon^{2} P, \quad \tau=\varepsilon^{2} S,
\end{array}\right\}
$$

and find that to leading order (with $\nu, \varepsilon, \bar{\delta} \ll 1$ )

$$
\left.\begin{array}{c}
-s^{\prime} \frac{\partial P}{\partial \zeta} \sim \frac{\partial T_{r z}}{\partial \zeta}, \quad \frac{\partial P}{\partial \zeta} \sim-\phi, \quad \eta \sim \frac{1}{S^{n-1}} \mathrm{e}^{-\phi}, \\
T_{r z} \sim \frac{\eta}{r} \frac{\partial^{2} \Psi}{\partial \zeta^{2}}, \quad T_{r r} \sim-\frac{2 \eta}{r} s^{\prime} \frac{\partial^{2} \Psi}{\partial \zeta^{2}}, \quad T_{z z} \sim \frac{2 \eta}{r} s^{\prime} \frac{\partial^{2} \Psi}{\partial \zeta^{2}}, \\
\varepsilon^{2} \phi_{t}-\varepsilon \dot{s} \phi_{\zeta}+\frac{\omega^{3}}{r}\left(\Psi_{\zeta} \phi_{r}-\Psi_{r} \phi_{\zeta}\right) \sim \phi_{\zeta \zeta}, \quad S \sim\left|T_{r z}\right|,
\end{array}\right\}
$$

where $s^{\prime}=\partial s / \partial r, \dot{s}=\partial s / \partial t$, and subscripts on variables other than stresses indicate partial derivatives.

The boundary conditions we need to apply are

$$
T_{r z}, P, \phi \rightarrow 0 \quad \text { as } \zeta \rightarrow \infty,
$$

which allows a matching to sub-lithospheric values, and

$$
\phi_{\zeta} \rightarrow \Gamma, \quad \Psi \rightarrow 0 \quad \text { as } \zeta \rightarrow-\infty,
$$

which matches the variables to the lithospheric values. In writing (A 30), we follow Fowler (1985), but in fact this detail of that paper is in error, because it neglects the existence of a passive 'outer' thermal boundary layer that is the continuation of the steady upwelling plume. In the present situation, we are perhaps more justified in neglecting this layer, as we are concerned with the impingement of an isolated plume on the lithosphere, and do not suppose that a steady circulation necessarily exists below it.

Fowler (1985) originally suggested another possible structure to the delamination layer, based on the idea that the lid base was relatively flat. He thought that this possibility was less likely, and in our subsequent work (Fowler 1993; Fowler \& O'Brien 1996), we have followed the approach suggested here, in which lid slope is significant. Fowler's (1985) study concerned Newtonian viscosity $(n=1)$, and his results appear consistent with numerical solutions (e.g. Moresi \& Solomatov 1995), but less so with non-Newtonian convection (Solomatov \& Moresi 1997). This issue remains unresolved. It seems unlikely that such a fundamental distinction would depend on the value of $n$, and it seems unlikely that numerical calculations alone can dictate the correct form of solution. On the other hand, it is also the case that certain features of the asymptotic structure of strongly variable viscosity convection are unclear; apart from the size of the lid slope, there is the comment of the preceding paragraph, and also the correct way to formulate boundary conditions for the lid slope equation (here, (A 36)). 
Table 3. The dependence of $B_{n}$ on $n$

\begin{tabular}{lc}
\hline$n$ & $B_{n}$ \\
\hline 1 & 0.087 \\
2 & $4.74 \times 10^{-3}$ \\
3 & $1.39 \times 10^{-4}$ \\
3.5 & $1.98 \times 10^{-5}$ \\
4 & $2.53 \times 10^{-6}$ \\
5 & $3.15 \times 10^{-8}$ \\
\hline
\end{tabular}

We can always neglect the small time derivative terms in the heat equation, but although $\omega^{3} \approx 0.5 \times 10^{-6}$, we must retain the convective terms, partly because $\Psi$ becomes large as $\zeta \rightarrow \infty$, and partly because the smallness of $\omega$ is offset by other factors, as we shall find below (see equation (B 9)). The above equations (A 29) then simplify to the following three:

$$
\left.\begin{array}{rl}
\frac{\partial T_{r z}}{\partial \zeta} & \sim s^{\prime} \phi, \\
\Psi_{\zeta \zeta} & \sim r\left|T_{r z}\right|^{n-1} T_{r z} \mathrm{e}^{\phi}, \\
\omega^{3}\left(\Psi_{\zeta} \phi_{r}-\Psi_{r} \phi_{\zeta}\right) & \sim r \phi_{\zeta \zeta} .
\end{array}\right\}
$$

These admit a physically appropriate similarity solution, in which

$$
\phi=g(\xi), \quad T_{r z}=\frac{s^{\prime}}{\Gamma} h(\xi), \quad \Psi=\frac{r s^{\prime}}{\Gamma^{3}}\left|\frac{s^{\prime}}{\Gamma}\right|^{n-1} f(\xi), \quad \eta=\left|\frac{\Gamma}{s^{\prime}}\right|^{n-1} N(\xi), \quad \xi=\Gamma \zeta,
$$

where

$$
\left.\begin{array}{rl}
h^{\prime} & =g, \\
f^{\prime \prime} & =|h|^{n-1} h \mathrm{e}^{g}, \\
g^{\prime \prime}+B_{n} f g^{\prime} & =0, \\
N & =\frac{1}{|h|^{n-1}} \mathrm{e}^{-G},
\end{array}\right\}
$$

and

$$
g^{\prime}(-\infty)=1, \quad f(-\infty)=0, \quad h(\infty)=0, \quad g(\infty)=0,
$$

providing that

$$
\frac{\omega^{3}}{r \Gamma}\left(\frac{r s^{\prime}}{\Gamma^{3}}\left|\frac{s^{\prime}}{\Gamma}\right|^{n-1}\right)^{\prime}=B_{n}
$$

a prime denoting differentiation with respect to $r$.

The equations (A 34) and (A 35) represent a generalization of the problem studied by Fowler (1985) for $n=1$, where it was found that $B_{1}=0.087$. We solve (A 34) and (A 35) by using a shooting method for

$$
\left.\begin{array}{rl}
H^{\prime} & =G, \\
F^{\prime \prime} & =|H|^{n-1} H \mathrm{e}^{G}, \\
G^{\prime \prime}+F G^{\prime} & =0,
\end{array}\right\}
$$




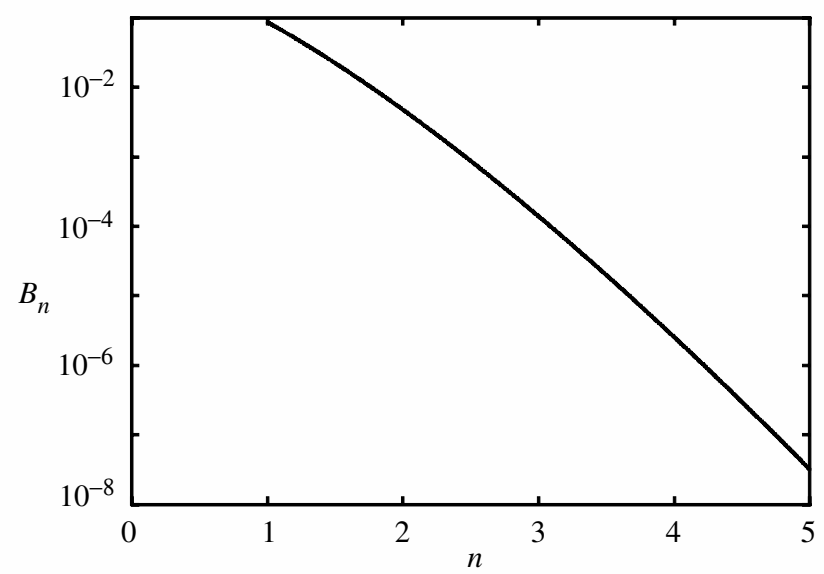

Figure 14. The dependence of $B_{n}$ on $n$.

with

$$
F=F^{\prime}=0, \quad G^{\prime}=c, \quad G=-c M, \quad H=H_{0}+\frac{1}{2} c M^{2} \quad \text { on } \xi=-M,
$$

where $M$ is taken to be indefinitely large (in practice, $M=20$ is satisfactory). We adjust $c$ and $H_{0}$ until $G$ and $H$ tend to zero at $\xi \rightarrow+\infty$. The solution of (A 34) is then

$$
g(\xi)=G(\xi / c), \quad h(\xi)=c H(\xi / c), \quad f(\xi)=c^{n+2} F(\xi / c),
$$

and $B$ is determined by

$$
B_{n}=\frac{1}{c^{n+3}}
$$

evidently, (A 34) with (A 35) is a nonlinear eigenvalue problem for $B_{n}$. Figure 14 (see also table 3 ) shows that $B_{n}$ depends strongly on $n$. It decreases roughly exponentially, as shown in the table. Given $B_{n}$, (A 36) determines the free boundary $s$ in (A 27), and in particular, we find that $s^{\prime}>0$, so that the modulus signs in (A 36) are redundant. The fact that $B_{n}$ is numerically small is a vindication of the assumption that $\omega$ is formally $O(1)$ in (A 32), since it is really the quantity $\beta=\left(B_{n} / \omega^{3}\right)^{1 / n}$ (see (B 9)) which is important.

\section{(d) Slab stress}

The heat equation (A 27) together with the free-boundary condition (A 36) determines the lithosphere base, providing there is rapid convection beneath. Apparently, (A 36) also requires specification of two boundary conditions for $s$ : we come back to this below.

In order to examine whether plastic flow occurs, we need to compute the stresses in the viscous lid; we refer to these as the slab stresses. As $\zeta \rightarrow-\infty$, we have from the delamination layer (assuming $s^{\prime}>0$ )

$$
\phi \sim \Gamma \zeta, \quad T_{r z} \sim \frac{1}{2} s^{\prime} \Gamma \zeta^{2}, \quad \Psi \sim \frac{r s^{\prime n}}{\Gamma^{n+2}}\left(\frac{1}{2} \Gamma^{2} \zeta^{2}\right)^{n} \mathrm{e}^{\Gamma \zeta},
$$


and thus back in the slab scales,

$$
\left.\begin{array}{rl}
\tau_{r z} & \sim \frac{1}{2} s^{\prime} \Gamma(z-s)^{2}, \\
\psi & \sim \frac{1}{\varepsilon^{2(n-1)}} \frac{r s^{\prime n}}{\Gamma^{n+2}}\left[\frac{1}{2} \Gamma^{2}(z-s)^{2}\right]^{n} \exp \left[\frac{\Gamma(z-s)}{\varepsilon}\right], \\
& \sim \frac{1}{\varepsilon^{2(n-1)}} \frac{r}{\Gamma^{2}} \tau_{r z}^{n} \exp \left(\frac{\theta}{\varepsilon}\right),
\end{array}\right\}
$$

as $z \rightarrow s$. Thus $\psi \sim 1 / \eta$, and this motivates the introduction of a modified stream function (also denoted $\Psi$, but not the same as in the delamination layer) by

$$
\psi=\varepsilon^{-2(n-1)} \Psi \mathrm{e}^{\theta / \varepsilon}, \quad \theta=1-(1 / T) .
$$

It follows that the stress/strain-rate constitutive relations are

$$
\left.\begin{array}{c}
\tau^{n-1} \tau_{r z}=\frac{1}{r}\left[\theta_{z}^{2} \Psi+\varepsilon\left\{2 \theta_{z} \Psi_{z}+\theta_{z z} \Psi\right\}+\varepsilon^{2} \Psi_{z z}\right] \\
-\nu^{2}\left[\frac{\theta_{r}^{2}}{r} \Psi+\varepsilon\left\{\frac{2 \theta_{r} \Psi_{r}}{r}+\Psi \frac{\partial}{\partial r}\left(\frac{\theta_{r}}{r}\right)\right\}+\varepsilon^{2} \frac{\partial}{\partial r}\left(\frac{\Psi_{r}}{r}\right)\right]-\delta^{*} \frac{\partial \tau_{r z}}{\partial t}, \\
\tau^{n-1} \tau_{r r}=2\left[\frac{\theta_{z} \theta_{r}}{r} \Psi+\varepsilon\left\{\frac{\theta_{r} \Psi_{z}}{r}+\frac{\partial}{\partial r}\left(\frac{\theta_{z} \Psi}{r}\right)\right\}+\varepsilon^{2} \frac{\partial}{\partial r}\left(\frac{\Psi_{z}}{r}\right)\right]-\delta^{*}\left(\frac{\partial \tau_{r r}}{\partial t}-\bar{c} \frac{\partial p}{\partial t}\right), \\
\tau^{n-1} \tau_{z z}=-2\left[\frac{\theta_{z} \theta_{r}}{r} \Psi+\frac{\varepsilon}{r}\left\{\theta_{z} \Psi_{r}+\theta_{r} \Psi_{z}+\theta_{z r} \Psi\right\}+\frac{\varepsilon^{2}}{r} \Psi_{z r}\right]-\delta^{*}\left(\frac{\partial \tau_{z z}}{\partial t}-\bar{c} \frac{\partial p}{\partial t}\right),
\end{array}\right\}
$$

where

$$
\delta^{*}=\bar{\delta} \varepsilon^{2 n} \mathrm{e}^{-\theta / \varepsilon}
$$

To leading order, (A 13) 1,2 give

$$
\frac{\partial p}{\partial r} \sim \frac{\partial \tau_{r z}}{\partial z}, \quad \frac{\partial p}{\partial z} \sim 1-T
$$

together with $p, \tau_{r z} \rightarrow 0$ on $z=s$ (from (A 42) and via (A 29), which implies

$$
\left.\begin{array}{rl}
p & \sim-\frac{1}{2} \Gamma(z-s)^{2}, \\
\tau_{r r} & \sim-s^{\prime 2} \Gamma(z-s)^{2}, \\
\tau_{z z} & \sim s^{\prime 2} \Gamma(z-s)^{2},
\end{array}\right\}
$$

as $z \rightarrow s)$. It follows that, on $z=q<s$,

$$
\tau_{r z}=\tau_{r z}^{\mathrm{visc}, q}=-\int_{q}^{s}(z-q) \frac{\partial T}{\partial r} \mathrm{~d} z, \quad p \sim p-\nu^{2} \tau_{z z} \sim-\int_{q}^{s}(1-T) \mathrm{d} z,
$$

and we assume that a plastic lid exists above $z=q$ (i.e. in $z<q$ ); this assumption is validated below when we calculate $q$.

We see now that the assumption of no elastic core corresponds to the assumption that $\delta^{*} \ll 1$ at $z=q$ (where $-\theta$ is maximal). Specifically, we require that

$$
-\theta<\varepsilon \ln \left(1 / \bar{\delta} \varepsilon^{2 n}\right)
$$


at $z=q$, that is,

$$
\frac{1}{T_{q}}-1<\varepsilon \ln \left(1 / \bar{\delta} \varepsilon^{2 n}\right),
$$

where $T=T_{q}$ at $z=q$. For Venus, if $\varepsilon=0.027, \bar{\delta}=0.4 \times 10^{-2}, n=3.5$, then $\varepsilon \ln \left(1 / \bar{\delta} \varepsilon^{2 n}\right) \approx 0.83$, so that if $T_{\mathrm{a}}=1700 \mathrm{~K}$, then (A 50) corresponds dimensionally to $T_{q}>928 \mathrm{~K}$. Since the surface temperature is $c a .750 \mathrm{~K}$, we see that the assumption of no elastic core is very reasonable.

\section{(e) Plastic lid}

By definition, $z=q$ represents the lower boundary of the region of failure $0<z<$ $q$. Because of our assumption that there is no elastic core, the effective rheology is visco-plastic, and we still have to solve (A 13), except that the effective viscosity is now determined by a suitable yield criterion.

The two commonly used failure criteria in plasticity are the Tresca yield criterion and the Von Mises yield criterion. The former takes the dimensional form

$$
\frac{1}{2}\left(\sigma_{1}-\sigma_{3}\right)=\tau_{\mathrm{c}},
$$

where $\sigma_{1}$ and $\sigma_{3}$ are the maximum and minimum principal stresses. In the present case, the longitudinal stresses are $\sigma_{r r}, \sigma_{\theta \theta}$ and $\sigma_{z z}$, and the sole deviatoric stress is $\tau_{r \theta}$. We then find that the principal stresses are

$$
\left.\begin{array}{rl}
\sigma_{ \pm}^{\prime} & =\frac{1}{2}\left(\sigma_{r r}+\sigma_{z z}\right) \pm \frac{1}{2}\left[\left(\sigma_{r r}-\sigma_{z z}\right)^{2}+4 \tau_{r \theta}^{2}\right]^{1 / 2}, \\
\sigma_{\theta}^{\prime} & =\sigma_{\theta \theta},
\end{array}\right\}
$$

and the corresponding principal deviatoric stresses $\left(\sigma_{i}^{\prime}=-p+\tau_{i}^{\prime}\right)$ are

$$
\left.\begin{array}{rl}
\tau_{ \pm}^{\prime} & =\left(\tau_{r r}+\tau_{z z}\right) \pm \frac{1}{2}\left[\left(\tau_{r r}-\tau_{z z}\right)^{2}+4 \tau_{r \theta}^{2}\right]^{1 / 2}, \\
\tau_{\theta}^{\prime} & =\tau_{\theta \theta}=-\left(\tau_{r r}+\tau_{z z}\right) .
\end{array}\right\}
$$

Suppose for example that $\tau_{r r} \approx-\tau_{z z}$. Then $\tau_{+}^{\prime}>\tau_{\theta}^{\prime}>\tau_{-}^{\prime}$, and the Tresca yield criterion (A 51) is

$$
\left(\tau_{r r}-\tau_{z z}\right)^{2}+4 \tau_{r \theta}^{2}=4 \tau_{\mathrm{c}}^{2} .
$$

In contrast, the Von Mises yield criterion is

$$
\tau_{+}^{\prime 2}+\tau_{\theta}^{\prime 2}+\tau_{-}^{\prime 2}=2 \tau_{\mathrm{c}}^{2}
$$

where $\tau_{\mathrm{c}}$ is the yield stress. If $\tau_{r r} \approx-\tau_{z z}, \tau_{\theta}^{\prime} \approx 0$, then this is approximately given by

$$
\left(\tau_{r r}-\tau_{z z}\right)^{2}+4 \tau_{r \theta}^{2}=4 \tau_{c}^{2},
$$

and we see that in this case, the two yield criteria are identical. As we will indeed find that $\tau_{r r} \approx-\tau_{z z}$, it is convenient to work with the Von Mises criterion; in terms of the second invariant $\tau^{2}$, we write (A 55) as

$$
\tau=\tau_{\mathrm{c}} .
$$


The above discussion refers to dimensional quantities. If we now follow the successive scaling $\tau \sim \eta_{\mathrm{a}} \kappa / l^{2}$ (A 1), and rescaling $\tau \sim \nu^{2} R a^{*}$ (A 10), then we find that the yield criterion can be written as

$$
\tau=\frac{C}{\nu}
$$

where

$$
C=\frac{\tau_{\mathrm{c}}}{\alpha \rho_{\mathrm{a}} g T_{\mathrm{a}} d}
$$

(recall that $d=\nu l$ ); with previously adopted values, $C \approx 0.22$ for $\tau_{\mathrm{c}}=300$ bar.

In the plastic lid, we rescale $\tau_{r r}, \tau_{z z}$ as

$$
\tau_{r r}=\frac{1}{\nu^{2}} T_{r r}, \quad \tau_{z z}=\frac{1}{\nu^{2}} T_{z z}
$$

so that the yield criterion is

$$
\nu^{2} \tau_{r z}^{2}+\frac{1}{2}\left[T_{r r}^{2}+T_{z z}^{2}+\left(T_{r r}+T_{z z}\right)^{2}\right]=C^{2}
$$

note that we suppose $C^{2} \leqslant O(1)$ : evidently this is a realistic assumption.

The stress equations are (A 61), together with

$$
\left.\begin{array}{l}
\frac{\partial p}{\partial r}=\frac{\partial \tau_{r z}}{\partial z}+\frac{1}{r} \frac{\partial}{\partial r}\left(r T_{r r}\right)+\frac{T_{r r}+T_{z z}}{r}, \\
\frac{\partial p}{\partial z}=1-T+\frac{\partial T_{z z}}{\partial z}+\frac{\nu^{2}}{r} \frac{\partial}{\partial r}\left(r \tau_{r z}\right),
\end{array}\right\}
$$

and thus, to leading order,

$$
p-T_{z z}=\int_{0}^{z}(1-T) \mathrm{d} z+\Delta_{\mathrm{s}},
$$

where $\Delta_{\mathrm{S}}$ is the uplift introduced earlier in (A 26), which has to be determined. We require the normal stress at $z=q$ to be continuous, and it follows from $(\mathrm{A} 48)_{2}$ that the uplift is given by the isostatic result

$$
\Delta_{\mathrm{s}}=-\int_{0}^{s}(1-T) \mathrm{d} z .
$$

(Notice that the 'uplift' is negative: this simply refers to the level it is measured from.)

Now with $p$ given by (A 63), we have two equations (A 61) and (A 62) 1 for the variables $T_{r r}, T_{z z}$, and $\tau_{r z}$; a solution cannot be obtained without considering the constitutive relations for the stresses. With the rescaling (see (A 60)), these are (from $(\mathrm{A} 13)_{3-5}$, providing $\bar{\delta}$ is sufficiently small)

$$
\left.\begin{array}{rl}
\nu^{2}\left[\frac{\tau_{r z}}{\eta \varepsilon^{2} \nu^{2}}\right] & =\frac{1}{r} \psi_{z z}-\nu^{2} \frac{\partial}{\partial r}\left(\frac{1}{r} \frac{\partial \psi}{\partial r}\right), \\
{\left[\frac{T_{r r}}{\eta \varepsilon^{2} \nu^{2}}\right]} & =2 \frac{\partial}{\partial r}\left(\frac{1}{r} \frac{\partial \psi}{\partial z}\right), \\
{\left[\frac{T_{z z}}{\eta \varepsilon^{2} \nu^{2}}\right]} & =-\frac{2}{r} \frac{\partial^{2} \psi}{\partial z \partial r},
\end{array}\right\}
$$


and it follows from this that, in relative terms, $\psi_{z z} \ll \psi_{z r}$, or equivalently,

$$
\psi \sim r u_{\mathrm{s}} z\left[1+O\left(\nu^{2}\right)\right]
$$

where $u_{\mathrm{s}}(r)$ is a (very small) surface velocity, which is to be found. We then have

$$
\left.\begin{array}{l}
\frac{T_{r r}}{\eta \varepsilon^{2} \nu^{2}} \sim 2 u_{\mathrm{s}}^{\prime}, \\
\frac{T_{z z}}{\eta \varepsilon^{2} \nu^{2}} \sim-\frac{2}{r}\left(r u_{\mathrm{s}}\right)^{\prime},
\end{array}\right\}
$$

where a prime denotes differentiation with respect to $r$.

Below the plastic lid, (A 43) implies that the horizontal velocity is

$$
\psi_{z} \sim \varepsilon^{-(2 n-1)} \mathrm{e}^{\theta / \varepsilon} \Psi
$$

where $\Psi$ is given (if $\left.\delta^{*} \ll 1\right)$ from $(\mathrm{A} 44)$ by

$$
\Psi \approx \frac{r \tau_{r z}^{n}}{\theta_{z}^{2}},
$$

since $\tau \approx \tau_{r z}>0$. This suggests (and we corroborate below) that the horizontal velocity in the plastic lid is

$$
u_{\mathrm{s}}=\varepsilon^{-(2 n-1)} \mathrm{e}^{\theta_{q} / \varepsilon} U,
$$

where $U=O(1)$ and $\theta_{q}=\left.\theta\right|_{z=q}$. In that case

$$
u_{\mathrm{s}}^{\prime} \sim \frac{\theta_{q}^{\prime}}{\varepsilon} u_{\mathrm{s}} \gg u_{\mathrm{s}},
$$

and therefore (as we anticipated above)

$$
T_{r r} \approx-T_{z z} \approx 2 \eta \varepsilon \nu^{2} \theta_{q}^{\prime} u_{\mathrm{s}}
$$

Because of (A 72), (A 61) is, to leading order, simply

$$
T_{r r} \approx-C
$$

(the minus sign is chosen to give a sensible definition of $q$ below); therefore

$$
\eta=\frac{C \varepsilon^{2(n-1)} \mathrm{e}^{-\theta_{q} / \varepsilon}}{2 \nu^{2} \theta_{q}^{\prime}|U|},
$$

where we require $U<0$ (back flow) if $\theta_{q}^{\prime}>0$ as we anticipate. In particular, $\eta \lesssim \eta_{q}$ $\left(\eta_{q}=\eta\right.$ at $\left.z=q\right)$ throughout the plastic lid. Therefore, failure of the lithosphere requires simply that the viscosity at the 'hinge' $q$ reaches the value at the lithosphere base $z=s$. We see from (A 74) that this is equivalent to $\theta_{q}$ reaching a critical value.

Finally, from $(\mathrm{A} 62)_{1}$ with $T_{r r} \approx-C \approx-T_{z z}$,

$$
\frac{\partial \tau_{r z}}{\partial z} \approx \frac{\partial p}{\partial r}+\frac{C}{r}=\int_{z}^{s} \frac{\partial T}{\partial r} \mathrm{~d} z+\frac{C}{r},
$$

using (A 63) and (A 64). Therefore,

$$
\tau_{r z}=\int_{0}^{z} \int_{z^{\prime}}^{s} \frac{\partial T}{\partial r}\left(r, z^{\prime \prime}, t\right) \mathrm{d} z^{\prime \prime} \mathrm{d} z^{\prime}+\frac{C z}{r} .
$$




\section{(f) Plastic skin}

As a consequence of the calculations above, there is a jump in the shear stress at the boundary between plastic and viscous parts of the lid. The shear stress must jump rapidly in the vicinity of this boundary, and we call this region of rapid change the plastic 'skin' (of the lid). The plastic and viscous values at $z=q$ of the stresses are (in the plastic scales), from the results given above,

$$
\left.\begin{array}{ll}
T_{r r}^{\text {plas }} \approx-T_{z z}^{\text {plas }}=-C, & \tau_{r z}^{\text {plas }}=\int_{0}^{s} z T_{r} \mathrm{~d} z-\int_{q}^{s}(z-q) T_{r} \mathrm{~d} z+\frac{C q}{r}, \\
T_{r r}^{\mathrm{visc}} \approx-T_{z z}^{\mathrm{visc}} \approx 0, & \tau_{r z}^{\mathrm{visc}}=-\int_{q}^{s}(z-q) T_{r} \mathrm{~d} z .
\end{array}\right\}
$$

Note the different uses of subscripts $r: T_{r}=\partial T / \partial r$, whereas $T_{r r}$ is the radial component of the normal stress. The discontinuity in the shear stress requires consideration of a boundary layer near $z=q$, and we put

$$
z=q+\varepsilon \zeta, \quad \frac{\partial}{\partial z}=\frac{1}{\varepsilon} \frac{\partial}{\partial \zeta}, \quad \frac{\partial}{\partial r}=\frac{\partial}{\partial r}-\frac{q^{\prime}}{\varepsilon} \frac{\partial}{\partial \zeta},
$$

where $q^{\prime}=\partial q / \partial r$; to leading order the stress equations are then (with the plastic scaling)

$$
\left.\begin{array}{rl}
-q^{\prime} \frac{\partial p}{\partial \zeta} & =\frac{\partial \tau_{r z}}{\partial \zeta}-q^{\prime} \frac{\partial T_{r r}}{\partial \zeta}, \\
\frac{\partial p}{\partial \zeta} & =\frac{\partial T_{z z}}{\partial \zeta} .
\end{array}\right\}
$$

Eliminating $\partial p / \partial \zeta$ and integrating across the boundary layer, we find

$$
q^{\prime}\left[T_{r r}-T_{z z}\right]_{\mathrm{visc}}^{\text {plas }}=\left[\tau_{r z}\right]_{\mathrm{visc}}^{\text {plas }} \text {. }
$$

It follows from (A 77) that $q$ is given to leading order by

$$
q^{\prime}=-\frac{1}{2 C}\left[\int_{0}^{s} z T_{r} \mathrm{~d} z+\frac{C q}{r}\right] .
$$

It is because we anticipate $T_{r}<0$ that we require the negative sign in (A 73), in order that $q^{\prime}>0$ : this follows from the observation that (A 81) can be written in the form

$$
2\left(r^{1 / 2} q\right)^{\prime}=-\frac{r^{1 / 2}}{C} \int_{0}^{s} z T_{r} \mathrm{~d} z .
$$

The solution can be formally completed by calculation of $U$. As in Fowler (1985), this can be done by completion of the analysis of the plastic skin. Knowledge of $U$ is only necessary in evaluating the failure criterion for $\theta_{q}$ using (A 74), but is not necessary in evaluating $\theta_{q}$ to leading order, and we omit its calculation here.

\section{Appendix B.}

Our numerical method for solving equations (3.8) and (3.9) is as follows. We define the front-fixing coordinate

$$
\eta=\frac{z}{s(r, t)}
$$


so that $T(\eta, r, t)$ satisfies

$$
T_{t}-\frac{s_{t}}{s} \eta T_{\eta}=\frac{1}{s^{2}} T_{\eta \eta}+h
$$

with

$$
\left.\begin{array}{rl}
T=T_{0} & \text { on } \eta=0 \\
T=1 & \text { on } \eta=1
\end{array}\right\}
$$

and

$$
\Gamma=\left.\frac{1}{s} T_{\eta}\right|_{\eta=1} .
$$

We need an expression for $s_{t}$ in order to solve (B 2); this can be obtained by differentiating $T(s(r, t), r, t)=1$ with respect to $t$, which leads to

$$
s_{t}=\left.\frac{\left\{\left|T_{\eta \eta}\right| / s^{2}\right\}-h}{\Gamma}\right|_{\eta=1},
$$

as long as $\Gamma \neq 0$. We use this for the first time-step, but thereafter use a backward difference approximation. Suitable initial conditions are given by

$$
s=s_{00} \quad \text { at } t=0,
$$

corresponding to the growth of a conductive lithosphere, and the corresponding initial $T$ profile should be the solution of the Crank-Gupta problem with $\Gamma=0$. In practice, we choose a cubic profile

$$
T=1-s_{00} \Gamma_{00}(1-\eta)-\frac{1}{2} h s_{00}^{2}(1-\eta)^{2}-\left[1-T_{0}-\frac{1}{2} h s_{00}^{2}-s_{00} \Gamma_{00}\right](1-\eta)^{3},
$$

in order to satisfy (B 3) and (B 4) with $\Gamma=\Gamma_{00}$ (we choose a small value of $\Gamma_{00} \neq 0$ to avoid difficulties in prescribing (B 5)), and also so that $\left|T_{\eta \eta}\right| \sim h s_{00}^{2}$ (which is implied from (B 5) if $\Gamma \approx 0$ ).

Our algorithm is then as follows. Given the solution at time-step $j-1$, we step forward $T$ to time-step $j$. From this we have an estimate for $\Gamma$ in $r>0$. However, the temperature equation can be solved at $r=0$ using the free-boundary condition $T_{z}=\Gamma_{0}$ on $z=s$ (if $\Gamma_{0}$ is prescribed), or else it can be solved directly if $s_{0}$ is given in (4.2). In our simulations, we choose this latter condition. Next we solve (3.9) at timestep $j$, using the given (or computed) value of $s_{0}$ in (4.2) as a boundary condition, together with (4.1). To see how to do this, we write (3.9) as the pair of equations (with $W>0$ )

$$
\left.\begin{array}{rl}
\frac{\partial s}{\partial r} & =\beta \Gamma^{1+(2 / n)} W^{1 / n}, \\
\frac{\partial}{\partial r}[r W] & =r \Gamma,
\end{array}\right\}
$$

where

$$
\beta=\left(\frac{B_{n}}{\omega^{3}}\right)^{1 / n} .
$$

The value of $\beta$ for $n=3.5$ is 2.7. This finally justifies our retention of $\omega$ in the delamination layer (see discussion following (A 31)). We specifically assume that

$$
W=0 \quad \text { on } r=0,
$$


assuming $\Gamma$ is finite at $r=0$; hence $\partial s / \partial r=0$ there. From (B 8), we find that in fact

$$
W \sim \frac{1}{2} \Gamma_{0} r \quad \text { as } r \rightarrow 0
$$

and thus

$$
\left(\frac{\partial s}{\partial r}\right)^{n} \sim \frac{\beta^{n}}{2} \Gamma_{0}^{n+3} r \quad \text { as } r \rightarrow 0 .
$$

If we took $W \neq 0$ at $r=0$, then $W \sim 1 / r$ as $r \rightarrow 0$, and $s$ would have infinite slope there. Solution of (B 8) is now carried out with initial conditions (B 10) and (4.2). The solution of this problem gives us an improved estimate for $s$ (and thus $s_{t}$ ), and the step for $T$ can be iterated, then the $s$ equation again, until a prescribed tolerance is reached.

\section{References}

Brace, W. F. \& Kohlstedt, D. L. 1980 Limits on lithospheric stress imposed by laboratory experiments. J. Geophys. Res. 85, 6248-6252.

Byerlee, J. 1978 Friction of rocks. Pure Appl. Geophys. 116, 615-626.

Cloetingh, S. A. P. L., Wortel, M. J. R. \& Vlaar, N. J. 1982 Evolution of passive continental margins and initiation of subduction zones. Nature 297, 139-142.

Cloetingh, S. A. P. L., Wortel, M. J. R. \& Vlaar, N. J. 1989 On the initiation of subduction zones. Pure Appl. Geophys. 129, 7-25.

Davies, R., England, P., Parsons, B., Billiris, H., Paradissis, D. \& Veis, G. 1997 Geodetic strain of Greece in the interval 1892-1992. J. Geophys. Res. 102, 24 571-24588.

DeLaughter, J. E. \& Jurdy, D. M. 1999 Corona classification by evolutionary stage. Icarus 139, 81-92.

England, P. \& Molnar, P. 1991 Inferences of deviatoric stress in actively deforming belts from simple physical models. Phil. Trans. R. Soc. Lond. A 337, 151-164.

England, P. \& Molnar, P. 1997 Active deformation of Asia: from kinematics to dynamics. Science 278, 647-651.

Evans, B., Fredrich, J. T. \& Wong, T.-F. 1990 The brittle-ductile transition in rocks: recent experimental and theoretical progress. Geophysical Monograph vol. 56, pp. 1-20. Washington, DC: American Geophysical Union.

Fowler, A. C. 1985 Fast thermoviscous convection. Stud. Appl. Math. 72, 189-219.

Fowler, A. C. 1993 Boundary-layer theory and subduction. J. Geophys. Res. 98, 21 992-22 005.

Fowler, A. C. 1997 Mathematical models in the applied sciences. Cambridge University Press.

Fowler, A. C. \& O'Brien, S. B. G. 1996 A mechanism for episodic subduction on Venus. J. Geophys. Res. 101, 4755-4763.

Goetze, C. 1978 The mechanics of creep in olivine. Phil. Trans. R. Soc. Lond. A 288, 99-119.

Goetze, C. \& Evans, B. 1979 Stress and temperature in the bending lithosphere as constrained by experimental rock mechanics. Geophys. J. R. Astr. Soc. 59, 463-478.

Henry, C., Das, S. \& Woodhouse, J. H. 2000 The great March 25, 1998, Antarctic Plate earthquake: moment tensor and rupture history. J. Geophys. Res. 105, 16 097-16 118.

Herrick, R. R. 1994 Resurfacing history of Venus. Geology 22, 703-706.

Hill, R. 1950 The mathematical theory of plasticity. Oxford: Clarendon Press.

Hobbs, B. E. \& Ord, A. 1988 Plastic instabilities: implications for the origin of intermediate and deep focus earthquakes. J. Geophys. Res. 93, 10 521-10540. 
Howard, L. N. 1966 Convection at high Rayleigh number. In Proc. 11th Int. Cong. Appl. Mech. (ed. H. Görtler), pp. 1109-1115. Springer.

Jurdy, D. M. \& Stefanick, M. 1999 Correlation of Venus surface features and geoid. Icarus 139, 93-99.

Karato, S. \& Wong, T. 1995 Rock deformation: ductile and brittle. Rev. Geophys. 33, 451-457.

Karato, S. \& Wu, P. 1993 Rheology of the upper mantle: a synthesis. Science 260, 771-778.

Kemp, D. V. \& Stevenson, D. J. 1996 A tensile, flexural model for the initiation of subduction. Geophys. J. Int. 125, 73-94.

Kirby, S. H. 1977 State of stress in the lithosphere: inferences from the flow law of olivine. Pure Appl. Geophys. 115, 245-258.

Kirby, S. H. \& Kronenberg, A. K. 1987 Rheology of the lithosphere: selected topics. Rev. Geophys. 25, 1219-1244.

McKenzie, D. P. 1977 The initiation of trenches: a finite amplitude instability. In Island arcs, deep sea trenches, and back-arc basins (ed. M. Talwani \& W. C. Pitman III), pp. 57-61. Washington, DC: American Geophysical Union.

Mackwell, S. J., Zimmerman, M. E. \& Kohlstedt, D. L. 1998 High-temperature deformation of dry diabase with application to tectonics on Venus. J. Geophys. Res. 103, 975-984.

Moresi, L.-N. \& Solomatov, V. S. 1995 Numerical investigation of 2D convection with extremely large viscosity variations. Phys. Fluids 7, 2154-2162.

Morris, S. \& Canright, D. 1984 A boundary-layer analysis of Bénard convection in a fluid of strongly temperature-dependent viscosity. Phys. Earth Planet. Inter. 36, 355-373.

Mueller, S. \& Phillips, R. J. 1991 On the initiation of subduction. J. Geophys. Res. 96, 651-665.

Musser Jr, G. S. \& Squyres, S. W. 1997 A coupled thermal-mechanical model for corona formation on Venus. J. Geophys. Res. 102, 6581-6595.

Nataf, H.-C. \& Richter, F. M. 1982 Convection experiments in fluids with highly temperaturedependent viscosity and the thermal evolution of the planets. Phys. Earth Planet. Inter. 29, 320-329.

Nimmo, F. \& McKenzie, D. 1998 Volcanism and tectonics on Venus. A. Rev. Earth Planet. Sci. 26, 23-51.

Philips, R. J. \& Hansen, V. L. 1994 Tectonic and magmatic evolution of Venus. A. Rev. Earth Planet. Sci. 22, 597-654.

Reese, C. C., Solomatov, V. S. \& Moresi, L.-N. 1998 Heat transport efficiency for stagnant lid convection with dislocation viscosity: application to Mars and Venus. J. Geophys. Res. 103, $13643-13657$.

Reese, C. C., Solomatov, V. S. \& Moresi, L.-N. 1999 Non-Newtonian stagnant lid convection and magmatic resurfacing on Venus. Icarus 139, 67-80.

Sandwell, D. T. \& Schubert, G. 1992 Evidence for retrograde lithospheric subduction on Venus. Science 257, 766-770.

Schaber, G. G., Strom, R. G., Moore, H. J., Soderblom, L. A., Kirk, R. L., Chadwick, D. J., Dawson, D. D., Gaddis, L. R., Boyce, J. M. \& Russell, J. 1992 Geology and distribution of impact craters on Venus: what are they telling us? J. Geophys. Res. 97, 13 257-13 301.

Schubert, G., Moore, W. B. \& Sandwell, D. T. 1994 Gravity over coronae and chasmata on Venus. Icarus 112, 130-146.

Shemenda, A. I. 1992 Horizontal lithosphere compression and subduction: constraints provided by physical modelling. J. Geophys. Res. 17, $11097-11116$.

Smrekar, S. E. \& Stofan, E. R. 1997 Corona formation and heat loss on Venus by coupled upwelling and delamination. Science 277, 1289-1294.

Solomatov, V. S. \& Moresi, L.-N. 1996 Stagnant lid convection on Venus. J. Geophys. Res. 101, 4737-4753. 
Solomatov, V. S. \& Moresi, L.-N. 1997 Three regimes of mantle convection with non-Newtonian viscosity and stagnant lid convection on the terrestrial planets. Geophys. Res. Lett. 24, 19071910.

Solomon, S. C. \& Head, J. W. 1991 Fundamental issues in the geology and geophysics of Venus. Science 252, 252-260.

Solomon, S. C. (and 10 others) 1992 Venus tectonics: an overview of Magellan observations. $J$. Geophys. Res. 97, 13 199-13255.

Squyres, S. W., Janes, D. M., Baer, G., Bindschadler, D. L., Schubert, G., Sharpton, V. L. \& Stofan, E. R. 1992 The morphology and evolution of coronae on Venus. J. Geophys. Res. 97, 13611-13 634 .

Strom, R. G., Schaber, G. G. \& Dawson, D. D. 1994 The global resurfacing of Venus. J. Geophys. Res. 99, $10899-10926$.

Tozer, D. C. 1972 The present thermal state of the terrestrial planets. Phys. Earth Planet. Inter. 6, 182-197.

Tsenn, M. C. \& Carter, N. L. 1987 Upper limits of power law creep in rocks. Tectonophysics 136, 1-26.

Turcotte, D. L. 1993 An episodic hypothesis for Venusian tectonics. J. Geophys. Res. 98, 1706117068 .

Turcotte, D. L. \& Schubert, G. L. 1982 Geodynamics. Wiley.

Turcotte, D. L., Haxby, W. F. \& Ockendon, J. R. 1977 Lithospheric instabilities. In Island arcs, deep sea trenches, and back-arc basins (ed. M. Talwani \& W. C. Pitman III), pp. 63-69. Washington, DC: American Geophysical Union.

Von Herzen, R., Ruppel, C., Molnar, P., Nettles, M., Nagihara, S. \& Ekström, G. 2001 A constraint on the shear stress at the Pacific-Australian plate boundary from heat flow and seismicity at the Kermadec forearc. J. Geophys. Res. 106, 6817-6833.

Weinstein, S. A. \& Olson, P. L. 1992 Thermal convection with non-Newtonian plates. Geophys. J. Int. 111, 515-530. 